\title{
Investigation of the cosmic ray population and magnetic field strength in the halo of NGC $891^{\star}$
}

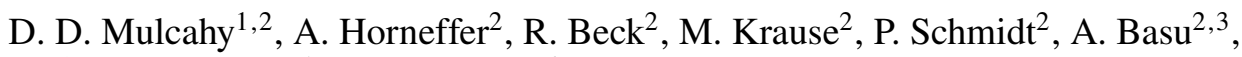 \\ K. T. Chyży ${ }^{4}$, R.-J. Dettmar ${ }^{5}$, M. Haverkorn ${ }^{6}$, G. Heald ${ }^{7}$, V. Heesen ${ }^{8}$, C. Horellou ${ }^{9}$, M. Iacobelli ${ }^{10}$, \\ B. Nikiel-Wroczyński ${ }^{4}$, R. Paladino ${ }^{11}$, A. M. M. Scaife ${ }^{1}$, Sarrvesh S. Sridhar ${ }^{10,12}$, R. G. Strom ${ }^{10,13}$, \\ F. S. Tabatabaei ${ }^{14,15}$, T. Cantwell ${ }^{1}$, S. H. Carey ${ }^{16}$, K. Grainge ${ }^{1}$, J. Hickish ${ }^{16}$, Y. Perrot ${ }^{16}$, N. Razavi-Ghods ${ }^{16}$, \\ P. Scott ${ }^{16}$, and D. Titterington ${ }^{16}$

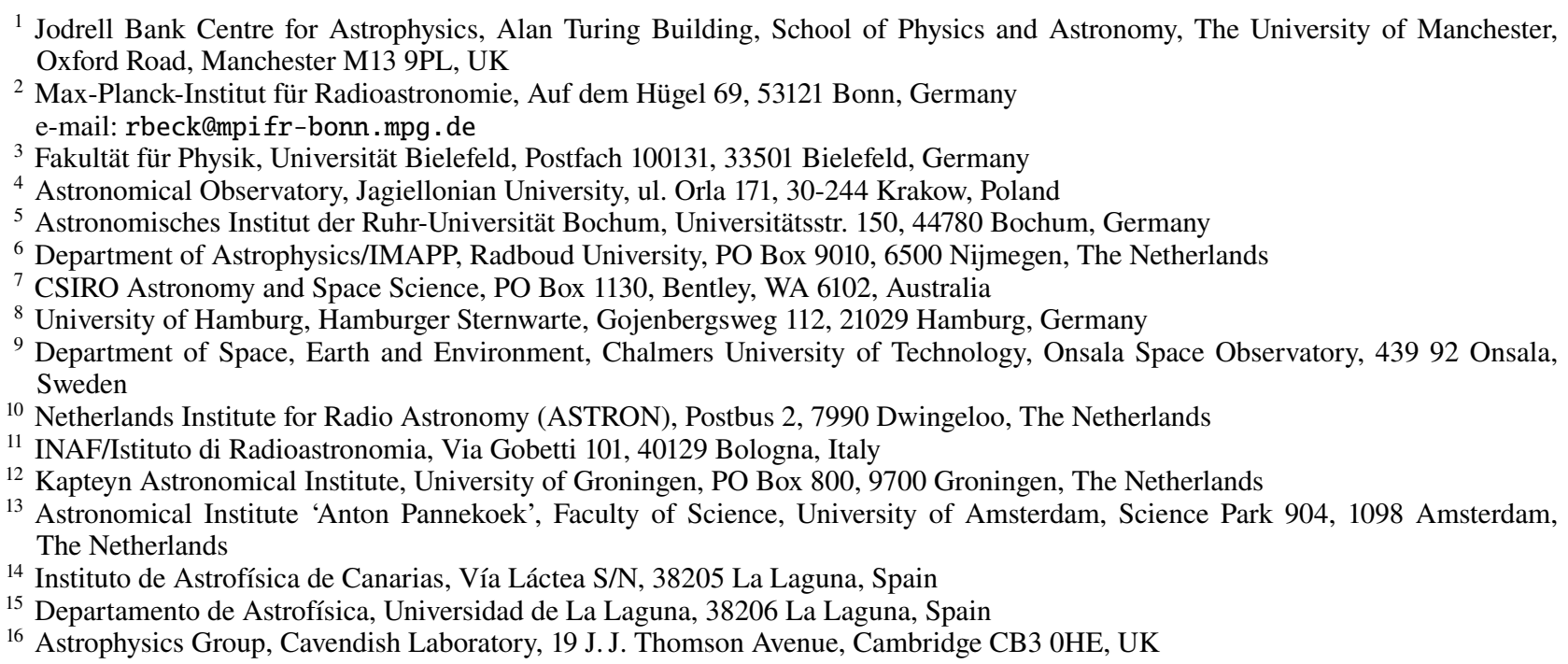

Received 15 February 2018 / Accepted 29 March 2018

\begin{abstract}
Context. Cosmic rays and magnetic fields play an important role for the formation and dynamics of gaseous halos of galaxies. Aims. Low-frequency radio continuum observations of edge-on galaxies are ideal to study cosmic-ray electrons (CREs) in halos via radio synchrotron emission and to measure magnetic field strengths. Spectral information can be used to test models of CRE propagation. Free-free absorption by ionized gas at low frequencies allows us to investigate the properties of the warm ionized medium in the disk.

Methods. We obtained new observations of the edge-on spiral galaxy NGC 891 at 129-163 MHz with the LOw Frequency ARray (LOFAR) and at 13-18 GHz with the Arcminute Microkelvin Imager (AMI) and combine them with recent high-resolution Very Large Array (VLA) observations at 1-2 GHz, enabling us to study the radio continuum emission over two orders of magnitude in frequency. Results. The spectrum of the integrated nonthermal flux density can be fitted by a power law with a spectral steepening towards higher frequencies or by a curved polynomial. Spectral flattening at low frequencies due to free-free absorption is detected in star-forming regions of the disk. The mean magnetic field strength in the halo is $7 \pm 2 \mu \mathrm{G}$. The scale heights of the nonthermal halo emission at $146 \mathrm{MHz}$ are larger than those at $1.5 \mathrm{GHz}$ everywhere, with a mean ratio of $1.7 \pm 0.3$, indicating that spectral ageing of CREs is important and that diffusive propagation dominates. The halo scale heights at $146 \mathrm{MHz}$ decrease with increasing magnetic field strengths which is a signature of dominating synchrotron losses of CREs. On the other hand, the spectral index between $146 \mathrm{MHz}$ and $1.5 \mathrm{GHz}$ linearly steepens from the disk to the halo, indicating that advection rather than diffusion is the dominating CRE transport process. This issue calls for refined modelling of CRE propagation.

Conclusions. Free-free absorption is probably important at and below about $150 \mathrm{MHz}$ in the disks of edge-on galaxies. To reliably separate the thermal and nonthermal emission components, to investigate spectral steepening due to CRE energy losses, and to measure magnetic field strengths in the disk and halo, wide frequency coverage and high spatial resolution are indispensable.
\end{abstract}

Key words. galaxies: halos - galaxies: individual: NGC 891 - galaxies: ISM - galaxies: magnetic fields - cosmic rays radio continuum: galaxies

\footnotetext{
* LOFAR and AMI images (FITS files) are only available at the CDS via anonymous ftp to cdsarc.u-strasbg.fr (130.79.128.5) or via http://cdsarc.u-strasbg.fr/viz-bin/qcat?]/A+A/615/A98
} 


\section{Introduction}

Magnetic fields and cosmic rays are dynamically relevant in the disks of spiral galaxies because the magnetic energy density is similar to the kinetic energy density of turbulence but larger than the thermal energy density (e.g. Beck 2015). Part of the energy input from supernova remnants goes into the acceleration of cosmic rays and into the amplification of turbulent magnetic fields. This scenario can explain the tight correlation between radio and far-infrared emission that holds for the integrated luminosities of galaxies as well as for the local intensities within galaxies (e.g. Tabatabaei et al. 2013, 2017; Heesen et al. 2014).

The physical relationships between the various components of the interstellar medium (ISM) are less understood in gaseous galaxy halos. Continuum emission from thick disks or halos is observed from the radio to the X-ray spectral ranges, as well as $\mathrm{H}$ I radio line emission of neutral hydrogen and optical emission lines of ionized gas. As sources in the disk probably provide most of the energy, warm and hot gas, cosmic rays, and magnetic fields have to be transported from the disk into the halo. The required pressure could be thermal or nonthermal (cosmic rays and magnetic fields). Possible transport mechanisms are a "galactic wind" with a velocity sufficient for escape (Uhlig et al. 2012), a "galactic fountain" with cold gas returning to the galaxy (Shapiro \& Field 1976; Fraternali 2017), or "chimney" outbreaks driven by hot superbubbles (Norman \& Ikeuchi 1989). Cosmic rays can propagate relative to the gas with a velocity limited to the Alfvén speed by the streaming instability (Kulsrud \& Pearce 1969) or can diffuse along or across the magnetic field lines (Buffie et al. 2013). Especially unclear is the role of magnetic fields in outflows. A turbulent field may be transported together with the gas in an outflow while an ordered field can support or suppress the outflow, depending on its strength and orientation. This is complicated further due to the interplay of the magnetic field with an inhomogeneous outflow in which isotropic turbulent fields are converted into anisotropic turbulent fields due to shear and compression, thus creating an ordered field in the halo (Elstner et al. 1995; Moss \& Sokoloff 2017).

Edge-on galaxies are ideal laboratories to study the disk-halo connection and to investigate the driving forces of outflows. The discovery of a huge radio halo around NGC 4631 by Ekers \& Sancisi (1977) revealed the importance of nonthermal processes. The theory of cosmic-ray propagation into the halos was developed in great detail (Lerche \& Schlickeiser 1981, 1982; Pohl \& Schlickeiser 1990), but comparisons with observations were inconclusive due to the limited quality of the radio data at that time.

Recently, this topic has been revived with high-quality radio spectral index maps becoming readily available. This is driven both by the advent of broadband correlators at existing interferometric telescopes such as the Australia Telescope Compact Array (ATCA) and the Very Large Array (VLA), operating at $\mathrm{GHz}$ frequencies, as well as the arrival of entirely new facilities such as the LOw Frequency ARray (LOFAR) that opens a hitherto unexplored window on radio halos at $\mathrm{MHz}$ frequencies. As a promising step, Heesen et al. (2016), using a 1D cosmic ray transport model SPINNAKER (SPectral INdex Numerical Analysis of $\mathrm{K}$ (c)osmic-ray Electron Radio-emission), were able to extract properties of the cosmic-ray propagation in the halo of two edgeon galaxies ${ }^{1}$. As a key result, they could show that the vertical profiles of the spectral index can be used to distinguish between advection- and diffusion-dominated halos, the latter representing the case of no significant outflows. Extending the sample

\footnotetext{
1 wWw.github.com/vheesen/Spinnaker
}

Table 1. Observational data of NGC 891.

\begin{tabular}{lc}
\hline \hline Morphology $^{a}$ & $\mathrm{Sb}$ \\
Position of the nucleus $^{b}$ & $\mathrm{RA}(\mathrm{J} 2000)=02^{\mathrm{h}} 22^{\mathrm{m}} 33^{\mathrm{s} .4}$ \\
& $\operatorname{Dec}(\mathrm{J} 2000)=+42^{\circ} 20^{\prime} 57^{\prime \prime}$ \\
Position angle of major axis $^{c}$ & $23^{\circ}\left(0^{\circ}\right.$ is north $)$ \\
Inclination of disk $^{d}$ & $89.8^{\circ}\left(0^{\circ}\right.$ is face on $)$ \\
Distance $^{a}$ & $9.5 \mathrm{Mpc}\left(1^{\prime \prime} \approx 46 \mathrm{pc}\right)$ \\
Star-formation rate $^{e}$ & $3.3 M_{\odot} \mathrm{yr}^{-1}$ \\
Total mass $^{c}$ & $1.4 \times 10^{11} M_{\odot}$ \\
\hline
\end{tabular}

References. ${ }^{(a)}$ van der Kruit \& Searle (1981). ${ }^{(b)}$ Vigotti et al. (1989). ${ }^{(c)}$ Oosterloo et al. (2007). ${ }^{(d)}$ Xilouris et al. (1999). ${ }^{(e)}$ Arshakian et al. (2011).

to 12 edge-on spiral and Magellanic-type galaxies, Krause et al. (2018) and Heesen et al. (2018a) showed that many halos are advection dominated with outflow speeds similar to the escape velocity, raising the possibility of cosmic ray-driven winds in them.

A prime target for such studies is NGC 891 that is a fairly nearby edge-on spiral galaxy. NGC 891 is similar to our own Milky Way in terms of optical luminosity (de Vaucouleurs et al. 1991), Hubble type (Sb; van der Kruit \& Searle 1981), and rotational velocity ( $225 \mathrm{~km} \mathrm{~s}^{-1}$; Rupen 1991), but has a high starformation rate $\left(3.3 M_{\odot} \mathrm{yr}^{-1}\right.$, Arshakian et al. 2011) compared to the Milky Way (the Galactic value is $1.66 \pm 0.20 M_{\odot} \mathrm{yr}^{-1}$; Licquia \& Newman 2014); this is in accordance with the presence of approximately twice the amount of molecular gas of the Milky Way, with the radial distribution of CO remarkably similar in both galaxies (Scoville et al. 1993). Due to its proximity and very high inclination, NGC 891 is an observational testing ground for the study of disk and halo interactions and the galactic halo. The physical parameters of NGC 891 are summarized in Table 1.

NGC 891 possesses a bright, well-studied halo and for which a plethora of ancillary data from various gas components is available. Rand et al. (1990) and Dettmar (1990) independently detected diffuse $\mathrm{H} \alpha$ emission from ionized gas up to $4 \mathrm{kpc}$ distance from the galaxy's plane with an exponential scale height of about $1 \mathrm{kpc}$. A huge halo of neutral atomic $\mathrm{H}$ i gas with up to $22 \mathrm{kpc}$ extent was observed by Oosterloo et al. (2007). Howk \& Savage (1997) detected prominent dust lanes emerging vertically into the halo of NGC 891 which could partly be associated with energetic processes connected to massive star formation in the disk. Sofue (1987) interpreted such dust lanes as tracers of vertical magnetic fields. Diffuse line emission from $\mathrm{CO}$ molecules is observed up to about $1 \mathrm{kpc}$ distance from the plane (Garcia-Burillo et al. 1992). Infrared emission from polycyclic aromatic hydrocarbons (PAH) particles and warm dust is detected to about $2.5 \mathrm{kpc}$ from the plane with similar scale heights of about $300 \mathrm{pc}$ (Whaley et al. 2009), excited by photons escaping from the disk. Emission from cold dust in the sub-millimetre range extends up to about $2 \mathrm{kpc}$ (Alton et al. 1998) and has a larger scale height than the warm dust. Diffuse X-ray emission from hot gas was found in the halo up to $4 \mathrm{kpc}$ from the plane (Bregman \& Pildis 1994; Hodges-Kluck \& Bregman 2013).

NGC 891 has also been extensively observed in radio continuum emission throughout the past few decades. The first extensive interferometric investigation in radio continuum at 610, 1412 and $4995 \mathrm{MHz}$ with the Westerbork Synthesis Radio Telescope (WSRT) by Allen et al. (1978) revealed a strong steepening 
of the spectral index in the halo, but this result was affected by missing large-scale emission in the $4995 \mathrm{MHz}$ image. Hummel et al. (1991) observed NGC 891 at $327 \mathrm{MHz}$ and $610 \mathrm{MHz}$ with the WSRT and at $1490 \mathrm{MHz}$ with the VLA. The spectral index between $610 \mathrm{MHz}$ and $1.49 \mathrm{GHz}$ with a resolution of $40^{\prime \prime}$ showed that the inner and outer radio disks (at small and large distances from the centre) have significantly different spectra, partly due to the larger thermal fraction in the inner disk. The spectral steepening towards the halo is mild on the eastern side but steep on the western side. The radio disk at $1490 \mathrm{MHz}$ was described by an exponential scale height of $1.2 \mathrm{kpc}$ (scaled to the distance adopted in this paper, see Table 1). Beck et al. (1979) and Klein et al. (1984) observed NGC 891 at $8.7 \mathrm{GHz}$ and $10.7 \mathrm{GHz}$ with the $100-\mathrm{m}$ Effelsberg telescope. When comparing their data to those at $610 \mathrm{MHz}$, they found only a mild steepening of the spectral index ${ }^{2}$ from $\alpha \approx-0.75$ in the disk to about -0.9 in the halo and no further steepening until up to $6 \mathrm{kpc}$ above the galaxy's plane (scaled to the distance to NGC 891 adopted in this paper). The spectral index in the halo of NGC 891 allowed for the first time a comparison with CRE propagation models. The models of diffusion and advection by Strong (1978) predicted almost linear gradients of spectral index which were in conflict with the observations by Allen et al. (1978), but consistent with those by Beck et al. (1979) and Klein et al. (1984). Dumke et al. (1995) observed a sample of edge-on galaxies, including NGC 891, with the Effelsberg telescope at $10.55 \mathrm{GHz}$. The vertical profile was described by two exponential scale heights of $270 \mathrm{pc}$ and $1.8 \mathrm{kpc}$ for the disk and halo, respectively (Krause 2012).

The structure of the magnetic field in the halo of NGC 891 has been investigated through measurement of linearly polarized radio synchrotron emission. The aforementioned observations of Dumke et al. (1995) with the Effelsberg telescope at $10.55 \mathrm{GHz}$ revealed diffuse polarized emission from the disk with an orientation predominately parallel to the plane. Such a field structure is to be expected from magnetic field amplification by the action of a mean-field $\alpha \Omega$-dynamo in the disk (e.g. Beck et al. 1996) or from a small-scale dynamo in a differentially rotating disk (Pakmor et al. 2014) or from shearing of an initially vertical field (Nixon et al. 2018). From Effelsberg observations of NGC 891 at $8.35 \mathrm{GHz}$, Krause (2009) showed that the large-scale orientation of the halo magnetic field in the sky plane appears to be "X-shaped". Such a field structure could arise from a mean-field dynamo including a galactic wind (Moss et al. 2010) or from a helical field generated by a velocity lag of the rotating halo gas (Henriksen \& Irwin 2016). With the aid of Faraday rotation measures, Krause (2009) also found indication of a large-scale regular magnetic field within the disk of NGC 891, likely part of a spiral magnetic field.

Israel \& Mahoney (1990) found that the integrated flux densities of 68 galaxies at $57.5 \mathrm{MHz}$ are systematically below the extrapolation from measurements at $1.4 \mathrm{GHz}$ if one assumes a power-law spectrum with a constant slope. They also reported that the $57.5 \mathrm{MHz}$ flux density is a function of inclination angle of the disk with respect to the sky plane, with lower flux densities observed for galaxies with larger inclination angles which they interpreted as increasing free-free absorption caused by a clumpy medium with an electron temperature of $T_{\mathrm{e}} \approx 1000 \mathrm{~K}$ and an electron density of order $1 \mathrm{~cm}^{-3}$. However, no direct observational evidence of such a medium exists, not even in our own Galaxy. Hummel (1991), re-analyzing the data of Israel \& Mahoney (1990), and Marvil et al. (2015) observed a flattening in the integrated spectra of nearby galaxies, but

$2 I_{v} \propto v^{\alpha}$ where $I_{v}$ is the intensity at frequency $v$. found no dependence on inclination; this makes it less likely that free-free absorption is the cause of this flattening. Therefore, low-frequency observations of nearby galaxies, specifically of edge-on galaxies, need to be performed to clear up some of these contradictions.

The LOw Frequency ARray (LOFAR; van Haarlem et al. 2013) opened a new era of studying the diffuse, extended radio continuum emission in halos of nearby galaxies and their magnetic fields - the study of which has so far been hampered at $\mathrm{GHz}$ frequencies by spectral ageing of CREs. The range of short baselines of the High Band Antenna (HBA) Array allow the detection of extended emission from nearby galaxies. To date, results on two few star-forming low-inclination galaxies with LOFAR were published (Mulcahy et al. 2014; Heesen et al. 2018b), but the nature of halos around edge-on spiral galaxies still needs to be investigated at low radio frequencies.

In this paper, we present and analyse the first LOFAR observations of NGC 891 with the HBA array at a central frequency of $146 \mathrm{MHz}$. These data are complemented by observations from the Arcminute MicroKelvin Imager (AMI) at $15.5 \mathrm{GHz}$, thus providing us with two decades of radio frequency coverage. This paper is organized as follows: In Sects. 2 and 3, we describe the observational setup of the LOFAR and AMI observations along with the data reduction and imaging process. In Sect. 4, we present the maps of NGC 891 at both $146 \mathrm{MHz}$ and $15.5 \mathrm{GHz}$; this is followed by a discussion in Sect. 5 on the morphology of the galaxy in comparison with other wavelengths; here, we pay particular attention to broadband observations with the VLA at central frequencies of 1.5 and $6 \mathrm{GHz}$ from the CHANG-ES survey (Continuum Halos in Nearby Galaxies: An EVLA Survey; Irwin et al. 2012). In Sect. 6, we present the separation of thermal and nonthermal emission components, discuss the spectrum of the integrated nonthermal emission, and present maps of the total and nonthermal radio spectral indices. Estimates of the magnetic field strength are given in Sect. 7. In Sect. 8, we measure the scale heights of the nonthermal emission in the halo at different distances along the projected major axis of the galaxy. We discuss the implications of our findings in Sect. 9: free-free absorption, energy loss processes of CREs, and CRE propagation. A summary is given in Sect. 10.

\section{LOFAR observations and data processing}

\subsection{Observational setup and data selection}

The observations of NGC 891 were done in interleaved mode, switching between scans on the calibrator 3C48 (at RA(J2000) $\left.=01^{\mathrm{h}} 34^{\mathrm{m}} 41^{\mathrm{s}} 3, \operatorname{Dec}(\mathrm{J} 2000)=+33^{\circ} 09^{\prime} 35^{\prime \prime}\right)$ and the target NGC 891. A total of 44 stations were used for this observation, of which 32 were core stations and 12 were remote stations. Full details of the observational setup are shown in Table 2 .

Two of the scans on the target were not recorded properly and one had excessive radio-frequency interference (RFI), so that these scans were discarded after pre-processing. After subtracting overheads for the calibrator observations and for the switching of the beam positions, the remaining on-source integration time was $5.6 \mathrm{~h}$. During facet calibration (see Sect. 2.2.2), the self-calibration solutions of the calibrator for the target facet, containing NGC 891, did not converge for frequencies $>163.6 \mathrm{MHz}$, so those data were excluded as well. Hence, the resulting bandwidth that was used for the imaging was 34.4 MHz. Finally, the automatic flagging in the pipelines flagged a further $15 \%$ of the visibilities, mostly data affected by 

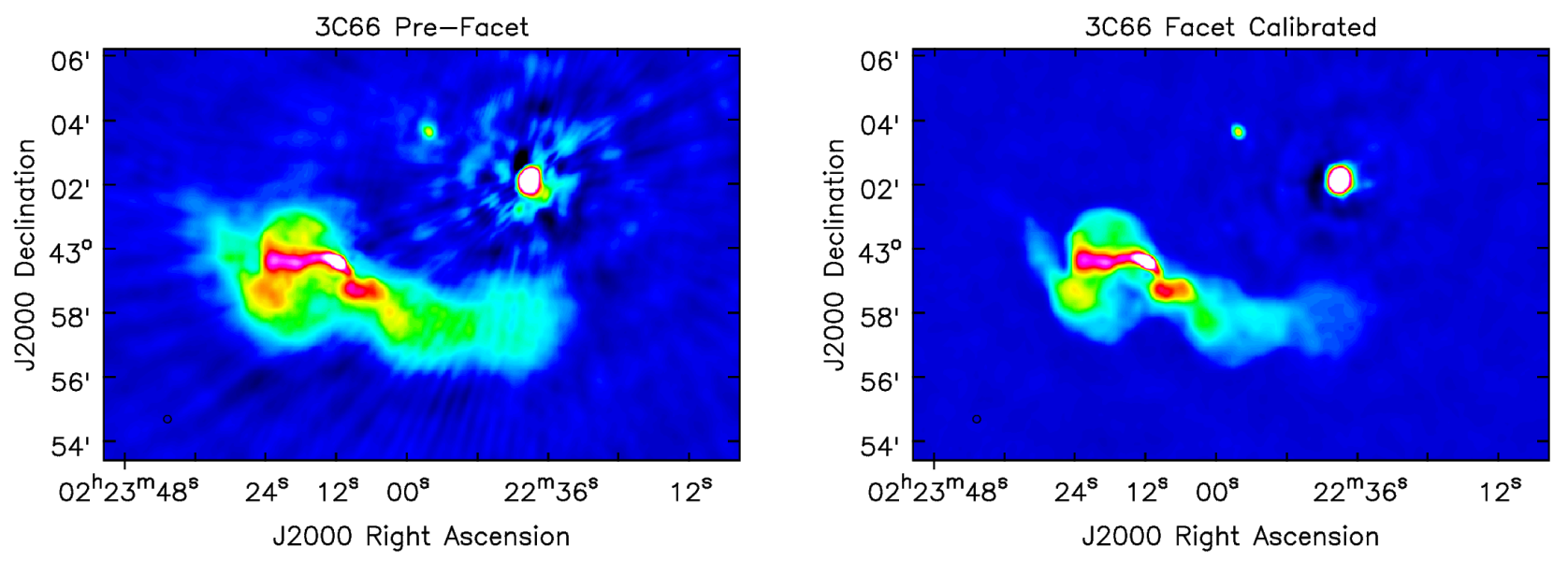

Fig. 1. Image of the compact source 3C66 A and the extended source 3C66 B after the initial direction-independent calibration (left panel) and after the direction-dependent facet calibration (right panel). Both images are smoothed to the same resolution of 14" and are displayed on the same colour scale. The strong artefacts around 3C66 A after the direction-independent calibration in the left map are caused by the calibration not being adequate for this direction. After calibrating specifically for this direction these artifacts are mostly gone.

Table 2. Parameters of the NGC 891 LOFAR HBA observations.

\begin{tabular}{lc}
\hline \hline Start date (UTC) & 31 March 2013 / 09:00 \\
End date (UTC) & 31 March 2013 / 16:59 \\
Interleaved calibrator & $3 \mathrm{C} 48$ \\
Scan length on calibrator & $2 \mathrm{~min}$ \\
Scan length on target & $15.8 \mathrm{~min}$ \\
Duration of observations & $8 \mathrm{~h}$ \\
Final time on target & $5.56 \mathrm{~h}(21 \mathrm{scans})$ \\
\hline Frequency range & $129.2-176.8 \mathrm{MHz}$ \\
Final frequency range & $129.2-163.6 \mathrm{MHz}$ \\
Total bandwidth on target & $47.6 \mathrm{MHz}$ \\
Final bandwidth on target & $34.4 \mathrm{MHz}$ \\
Reference frequency & $146.38 \mathrm{MHz}$ \\
\hline
\end{tabular}

RFI that were identified as spikes when plotting amplitudes as function of time or frequency.

Most of the short baselines between the "ears" of the core stations were flagged to avoid cross-talk. The remaining shortest baselines between the core stations of about $50 \mathrm{~m}$ ensure the detection of large diffuse emission on scales of up to $2^{\circ}$.

\subsection{Calibration}

Low-frequency, wide-field imaging data are subject to several effects that are usually negligible at higher frequencies, most notably distortions of the Earth's ionosphere and artifacts caused by the large number of bright background sources. In order to overcome these difficulties, we applied the novel technique of facet calibration. This method uses three main steps. First, a direction-independent calibration of the data with the prefactor pipeline that applies the flux density scale, corrects the Faraday rotation of the Earth's ionosphere, corrects for instrumental effects such as clock offsets between different LOFAR stations, and performs an initial round of phase calibration; second, the subtraction of all sources that are present in the supplied sky model with the Initial Subtract pipeline; last, the application of the Factor pipeline. This pipeline divides the field of view into a mosaic of smaller facets using Voronoi tesselation (e.g. Okabe et al. 2000).

\subsubsection{Initial calibration}

To calibrate our LOFAR dataset of NGC 891, we used a prototype version of the prefactor ${ }^{3}$ pipeline for the initial, direction-independent calibration and Version 1.0pre of Factor ${ }^{4}$ for the direction-dependent facet calibration.

The data of the calibrator and target were first pre-processed with the New Default Pre-Processing Pipeline (NDPPP) that includes RFI excision with aoflagger (Offringa et al. 2010, 2012), removal of edge-channels, and averaging to $4 \mathrm{~s}$ time- and $49 \mathrm{kHz}$ (4 channels per subband) frequency-resolution.

The data of the calibrator observations were then calibrated against a known model characterizing the detailed frequency dependence of the flux density of 3C48 (Scaife \& Heald 2012). This model thus sets the flux density scale of our data. The gain solutions from this calibration were then used to extract instrumental calibration parameters: gain amplitudes, station clock delays, and phase offsets between the $X$ - and $Y$-dipoles within a station.

These solutions were copied to the target data in order to correct for instrumental effects. To deal with the effects of strong off-axis sources, we predicted the visibilities of the four strongest sources (Cas A, Cyg A, Tau A, and Vir A) and flagged all visibilities to which they contributed more than an apparent (not corrected for primary-beam attenuation) flux density of $5 \mathrm{Jy}^{5}$. Then the data were averaged to the final resolution of $8 \mathrm{~s}$ and $98 \mathrm{kHz}$ ( 2 channels per subband) and concatenated into groups of 11 subbands to form 16 bands with $2.15 \mathrm{MHz}$ total bandwidth each. This resolution is sufficient to avoid decorrelation due to rapid changes in the ionospheric phase, while keeping the data volume manageable at 14.5 GByte per band. An additional round of automatic RFI excision with aoflagger was then performed on the concatenated data, because the algorithm is more effective in detecting RFI in data sets with wide bandwidths as compared with single subbands. As a final step for the direction-independent calibration, the data were phase calibrated

\footnotetext{
3 https://github.com/lofar-astron/prefactor

4 https://github.com/lofar-astron/factor

5 This threshold was found to be the best compromise between flagging too much data and thus increasing the rms noise and flagging too few data and thus not removing the residual sidelobes of these sources (van Weeren, priv. comm.).
} 


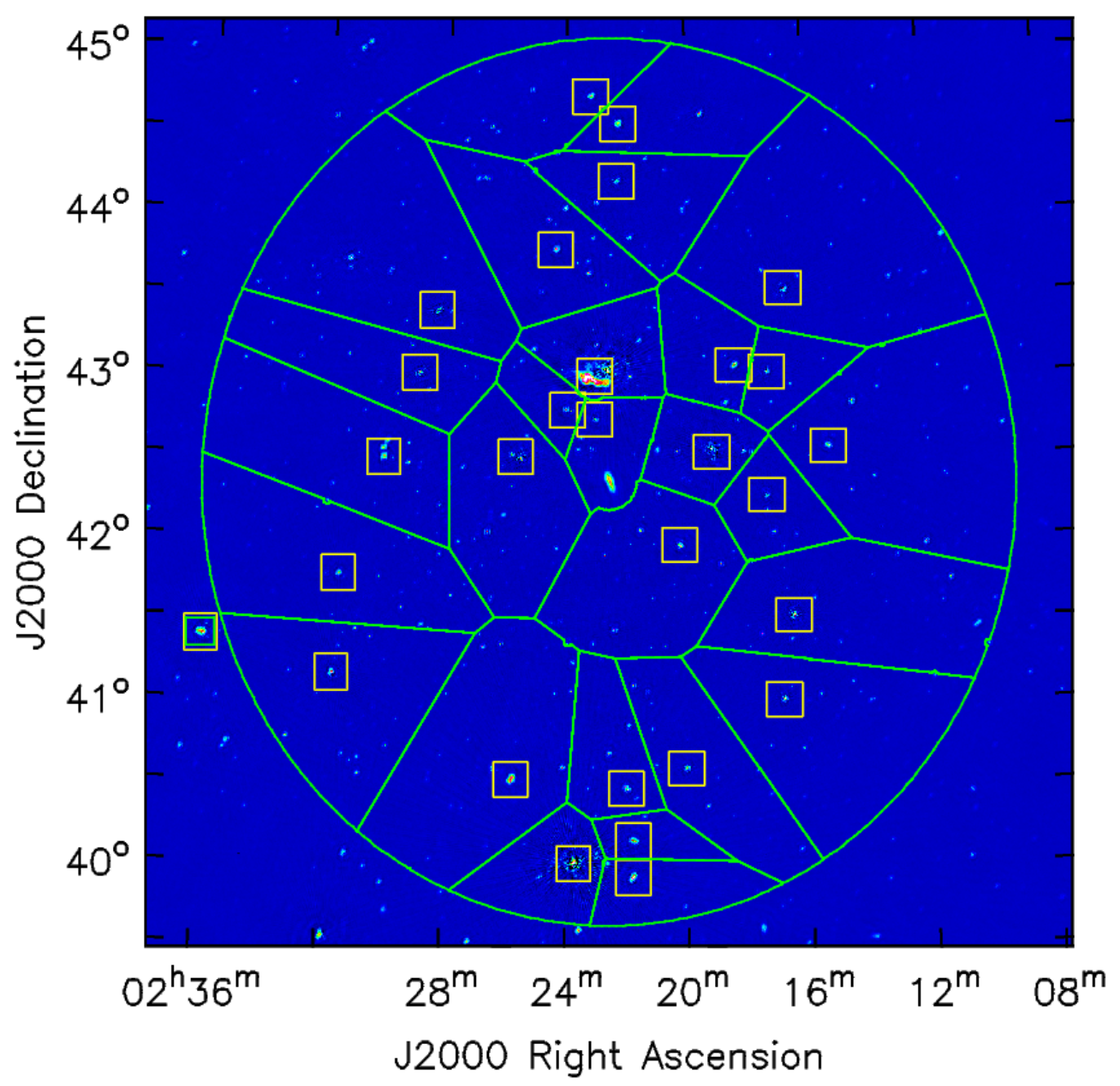

Fig. 2. Image of the full FoV of the LOFAR observations after initial calibration, with NGC 891 in the centre, 3C66 A/B above the centre, and 3C65 at the lower edge of the image. Overlaid is the faceting scheme that was used for the facet calibration: in orange the squares of the calibrator regions that were used to calibrate the facets and in green the resulting facets that are defined by Voronoi tessellation around the calibrators.

on a model generated from the LOFAR Global Sky Model (GSM; van Haarlem et al. 2013).

Finally, the 16 bands were imaged separately, first at a medium resolution (outer $u v$ cut at $7 \mathrm{k} \lambda$, about $20^{\prime \prime}$ resolution) then - after subtracting the sources found in the medium resolution images - at a lower resolution (outer $u v$ cut at $2 \mathrm{k} \lambda$, about $1.5^{\prime}$ resolution). The field of view (FoV) of the medium resolution images was 2.5 times the full width to half maximum (FWHM) of the station beam (between $9.4^{\circ}$ and $12.8^{\circ}$ depending on the frequency of the band) and the FoV of the low-resolution images 6.5 times the FWHM of the station beam $\left(24.5^{\circ}-33.3^{\circ}\right)$. The reason to create different kinds of images is to pick up low surface-brightness emission and to be able to image a larger FoV without prohibitive computing requirements. The final result is the combined list of sources from both imaging steps, the residual visibilities in which all detected sources have been subtracted, and the phase solutions from the last calibration step, for each of the 16 bands.

\subsubsection{Facet calibration}

The first step of the facet calibration is the selection of the calibration directions that contain the facet calibrators. For this step and for what follows we used the Factor pipeline that automates most of the necessary steps. In the first step, Factor uses the sky model for the highest frequency band and searches for strong and compact sources. This was done with the following selection parameters:

- maximum size of a single source: $2^{\prime}$;

- minimum apparent flux density of a single source: $100 \mathrm{mJy}$;

- maximum distance of single source to be grouped into one calibrator region: $6^{\prime}$;

- minimum total apparent flux density of the sources of a calibrator region: $250 \mathrm{mJy}$.

The resulting list of calibration directions was then manually modified in order to tailor it to our specific requirements. Apart from removing some of the weaker calibrators that were too close to each other, we also experimented how to define best the calibrator region around 3C66 (Fig. 1) - by far the brightest source in our FoV. In the end we decided to fully include both 3C66 A and 3C66 B into the region: while the extended emission of $3 \mathrm{C} 66 \mathrm{~B}$ is not suited for calibration, there is enough compact flux density in 3C66 A to allow for good calibration solutions even on the long baselines, whereas excluding $3 \mathrm{C} 66 \mathrm{~B}$ made calibration worse, probably because the short baselines could not be calibrated well as the sky model did not include the extended emission.

The 29 calibration directions after our manual adjustments are shown in Fig. 2. The facets are set up by Voronoi tesselation around the calibration direction up to a maximum 
radius of $2.5^{\circ}$ in $\mathrm{RA}$ and $2.7^{\circ}$ in $\mathrm{Dec}^{6}$ around the pointing centre. The facet boundaries are slightly deflected to avoid intersecting with detected sources in the sky model. We defined a region with a radius of $10.2^{\prime}$ around NGC 891 as the target facet, so that sources, as well as our target, are not split between two facets. The resulting facet boundaries are shown as lines in Fig. 2. The one direction outside the faceting radius (see the orange box outside of the green ellipse in Fig. 2) has only a calibration region but no facet associated with it which means that only a small area around the calibrator is imaged.

The core of Factor is the direction-dependent calibration. For each direction the residual visibilities are phase shifted to the direction of the calibration region and the sources within that region are added back to the visibilities. Then these data are self-calibrated, starting with the direction-independent calibration. The self-calibration has two loops: in the first loop, Factor only solves for a fast phase term to track the ionospheric delay; in the second loop, Factor solves again for this fast phase term and simultaneously for a slowly varying gain (phase + amplitude) term to also correct for residual effects from discrepancies in the beam model and from any other causes. After the calibration region has been calibrated, the full facet is imaged again: the visibilities are prepared in a similar fashion as they were for self-calibration, except that all sources within the facet are added back in and that the data are corrected with the self-calibration solutions. From this image an updated sky model for the facet is created which, together with the calibration solutions for this direction, is used to update the residual visibilities by subtracting the difference between the new and the original model. The following directions are then processed with the improved residual visibilities. This way the strong sources can be subtracted first and relatively weak sources can be used as calibrators.

We only processed one direction at a time, starting with 3C66 which has the largest apparent flux density and thus the highest signal-to-noise ratio, proceeding down in apparent flux density. Ordered in this way, the region that contains NGC 891 is in the 12th facet. Before processing the target facet, the directly adjacent facets were processed, too. The remaining facets contribute only little noise to the region around NGC 891, so we stopped processing there. The visibilities we used for the final imaging were the ones that Factor generated for the imaging of the full target facet; this means that all detected sources outside the target facet were subtracted, so that it was sufficient to image only the relatively small area of the target facet.

\subsection{Final imaging}

We used the Common Astronomy Software Applications ${ }^{7}$ (CASA; McMullin et al. 2007), Version 4.7, to image the facet containing NGC 891. Whilst CASA does not implement the LOFAR primary beam, NGC 891 is much smaller than the size of the LOFAR primary beam $\left(3.8^{\circ} \mathrm{FWHM}\right.$ at $\left.146 \mathrm{MHz}\right)$ and is located at the phase centre of our observation. Thus, systematic flux density errors due to the missing primary beam correction are minimal.

We created four different images. The first image, with no $u v$ taper applied and a robust weighting of -1.0 , achieves a high resolution of $8.3^{\prime \prime} \times 6.5^{\prime \prime}$, sufficient to resolve various features in the disk and the inner halo. Another version of this image was

\footnotetext{
6 The region is elliptical to account for the elongation of the LOFAR primary beam at lower elevations.

http://casa.nrao.edu
}

Table 3. Parameters of the NGC 891 AMI observations.

\begin{tabular}{lc}
\hline \hline 1st Small Array time (UTC) & 7-8 Dec. 2016 / 20:07-01:50 \\
2nd Small Array time (UTC) & $9-10 \mathrm{Dec} .2016 / 17: 23-01: 42$ \\
Large Array time (UTC) & 7 Dec. 2016 / 19:01-22:51 \\
Flux calibrators & 3C48 (LA); 3C286 (SA) \\
\hline Frequency range & $13-18 \mathrm{GHz}$ \\
Reference frequency & $14.5 \mathrm{GHz}$ \\
\hline
\end{tabular}

created by convolution to $12^{\prime \prime} \times 12^{\prime \prime}$. The third image has a moderate resolution, with an outer $u v$ taper of $7 \mathrm{k} \lambda$, a robust weighting of 0 , and convolved to $20^{\prime \prime} \times 20^{\prime \prime}$. The fourth, our low-resolution image, with an outer $u v$ taper of $2 \mathrm{k} \lambda$ and a robust weighting of +1.0 , is best suited to detect the low surface brightness of the extended halo.

For all images, we performed multi-scale and multifrequency synthesis CLEAN (Högbom 1974; Cornwell 2008; Rau \& Cornwell 2011) on the facet containing NGC 891, for which we used CLEAN manually drawn masks. The imaging parameters are given in Table 4.

\section{AMI observations and data processing}

\subsection{Observational setup}

The Arcminute Microkelvin Imager (AMI) telescope (Zwart et al. 2008) consists of two radio arrays, the Small Array (SA) and the Large Array (LA), located at the Mullard Radio Astronomy Observatory (Cambridge, UK). The SA is a compact array of ten 3.7-m paraboloid dishes and is sensitive to structures on angular scales between $2^{\prime}$ and $10^{\prime}$. The LA is an array of eight $12.8-\mathrm{m}$ dishes and is sensitive to scales between $0.5^{\prime}$ and $3^{\prime}$. Each array observes at a frequency range of $13-18 \mathrm{GHz}$ with 4096 channels split into two bands (Hickish et al. 2018).

The SA data were taken as a single pointing with interleaved observations of the phase calibrator J0222+4302 and 3C286 used as a flux density and bandpass calibrator. The LA data were taken as a mosaic, consisting of seven pointings arranged on a hexagonal raster centred on NGC 891. Individual pointings were cycled between the pointing centers every $60 \mathrm{~s}$, switching to the phase calibrator J0222+4302 every $10 \mathrm{~min}$ for $2 \mathrm{~min}$. Details of these observations are given in Table 3 .

\subsection{Calibration and imaging}

Calibration and imaging of the visibilities from both arrays were carried out using CASA. The first step, in calibrating AMI data, is the generation of the rain gauge correction which is a correction for the system temperature dependent on the weather during the observation. An initial round of flagging was carried out on the full resolution data, using the rflag option in CASA's flagdata task, in order to remove strong narrow-band RFI. The data were then averaged from 2048 to 64 channels for each band. We used the flux density scale of Perley \& Butler (2013) for calibrators 3C48 and 3C286. A tailored, in-house version of the CASA task setjy was used to correct for the fact that AMI measures single polarization $I+Q$. An initial round of phase only calibration was performed on the flux calibrator which was then used for delay and bandpass calibration. This was followed by another phase and amplitude calibration, applying the delay and bandpass solutions on the fly. The calibrated data were used for a second round of flagging, before performing a phase and amplitude calibration on the phase 

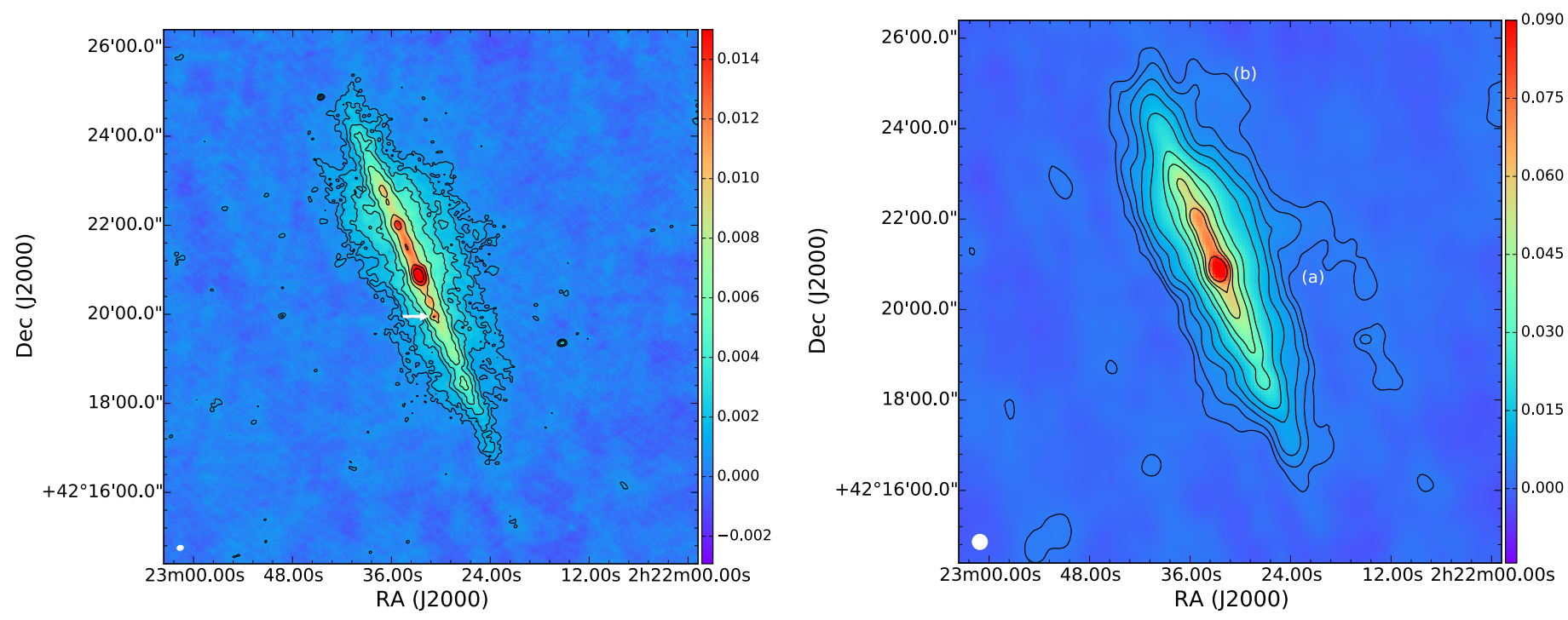

Fig. 3. LOFAR maps of NGC 891 observed at a central frequency of $146.4 \mathrm{MHz}$ with a bandwidth of $34.4 \mathrm{MHz}$ at two different resolutions. Left panel: Resolution of $8.3^{\prime \prime} \times 6.5^{\prime \prime}$ (shown by the filled ellipse in the bottom left corner). Contours are at 3, 5, 8, 12, 18, 32, 44, 64 $\times \sigma$ where $\sigma=0.29 \mathrm{mJy}^{-}$beam $^{-1} 1$ is the rms noise level. The location of SN1986J is shown by the white arrow. Right panel: Resolution of $20^{\prime \prime} \times 20^{\prime \prime}$ (shown by the filled ellipse in the bottom left corner). Contours are at 3, 5, 8, 12, 18, 32, 44, 64, 76 $\times \sigma$ where $\sigma=0.8 \mathrm{mJy}^{-}$beam $^{-} 1$ is the rms noise level. The features denoted by "(a)" and "(b)" are discussed in Sect. 4. The colour scale is in units of Jy/beam.

Table 4. Imaging parameters for the LOFAR images.

\begin{tabular}{lccc}
\hline \hline & High-resolution image & Medium-resolution image & Low-resolution image \\
\hline$u v$ taper & - & $7 \mathrm{k} \lambda$ & $2 \mathrm{k} \lambda$ \\
Weighting & -1 & 0 & +1 \\
Angular resolution & $8.3^{\prime \prime} \times 6.5^{\prime \prime}\left(381 \times 299 \mathrm{pc}^{2}\right)$ & $20^{\prime \prime} \times 20^{\prime \prime}\left(920 \times 920 \mathrm{pc}^{2}\right)$ & $40.0^{\prime \prime} \times 35.7^{\prime \prime}\left(1.84 \times 1.64 \mathrm{kpc}^{2}\right)$ \\
Cell size & $1.0^{\prime \prime}$ & $3.0^{\prime \prime}$ & $4.0^{\prime \prime}$ \\
\hline
\end{tabular}

calibrator, applying again the delay and bandpass solutions on the fly. After the flux density of the phase calibrator was bootstrapped (e.g. Lepage \& Billard 1992) the calibration tables were applied to the target data which were then averaged to 8 channels for imaging.

The data from both arrays were imaged with multi-scale CLEAN (Cornwell 2008) and each image was cleaned interactively. The SA image with its single pointing was primary beam corrected using the task PBCOR in AIPS with the defined SA primary beam ${ }^{8}$. Each of the seven LA pointings were imaged separately and converted into FITS files. These images were combined into a mosaic using the AIPS task FLATN which was then corrected for attenuation by the LA primary beam.

\section{Results: NGC 891 at $146 \mathrm{MHz}$ and $15.5 \mathrm{GHz}$}

\subsection{The LOFAR images}

The images of NGC 891 at a central frequency of $146 \mathrm{MHz}$ are shown at the highest resolution $\left(8.3^{\prime \prime} \times 6.5^{\prime \prime}\right)$ in Fig. 3 (left), at medium resolution $\left(20^{\prime \prime} \times 20^{\prime \prime}\right)$ in Fig. 3 (right), and at low resolution $\left(40.0^{\prime \prime} \times 35.7^{\prime \prime}\right)$ in Fig. 4. The image characteristics are given in Table 4 . The image at $12^{\prime \prime} \times 12^{\prime \prime}$ resolution is shown in the composite of Fig. 18.

The mean root-mean-square ( $\mathrm{rms}$ ) noise at high resolution is approximately $\sigma \simeq 0.29 \mathrm{mJy}^{\text {beam }}{ }^{-1}$ next to NGC 891 and about

\footnotetext{
8 AIPS, the Astronomical Image Processing System, is free software available from NRAO.
}

$0.26 \mathrm{mJy} \mathrm{beam}^{-1}$ in a quiet region. The noise at medium res-

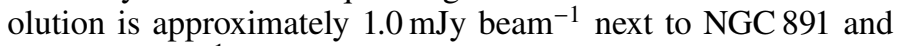
$0.8 \mathrm{mJy} \mathrm{beam}^{-1}$ in a quiet region.

The morphology of NGC 891 at $146 \mathrm{MHz}$ at low resolution is quite similar to that at higher frequencies $(327 \mathrm{MHz}$ and $610 \mathrm{MHz}$ ) as seen in Hummel et al. (1991). The radio halo bulges out in the northern sector of the galaxy. This is especially evident in the high-resolution image (Fig. 3 left) where diffuse emission extends to the north-east and north-west. It is known that the northern part of the disk has a larger star-formation rate than the southern part (Strickland et al. 2004).

Interesting extensions (marked "(a)" and "(b)" in Fig. 3) are seen in the western halo which were also observed at higher frequencies (Schmidt 2016, Schmidt et al. in prep.), but are more prominent at lower frequencies. Signs of feature "(a)" are also seen as low brightness emission in Fig. 4. These features are revisited later with information on their spectrum in Sect. 6.

The eastern radio halo of NGC 891 displays a "dumbbell" shape in Fig. 4, similar to what is observed on both sides of the halo of NGC 253 (Heesen et al. 2009). Such a shape can be a signature of dominating synchrotron losses (see Sect. 9.2). The two extensions on the eastern side of the galaxy out to approximately $5.5 \mathrm{kpc}$ from the major axis are similar to the ones found in NGC 5775 (Soida et al. 2011) and may be signs of outflows which eject gas from galaxies and enrich the local intergalactic medium. Between these two galactic spurs the radio emission extends out to about $4.5 \mathrm{kpc}$ from the plane. The maximum extent of the western halo of NGC 891 (outermost contour 


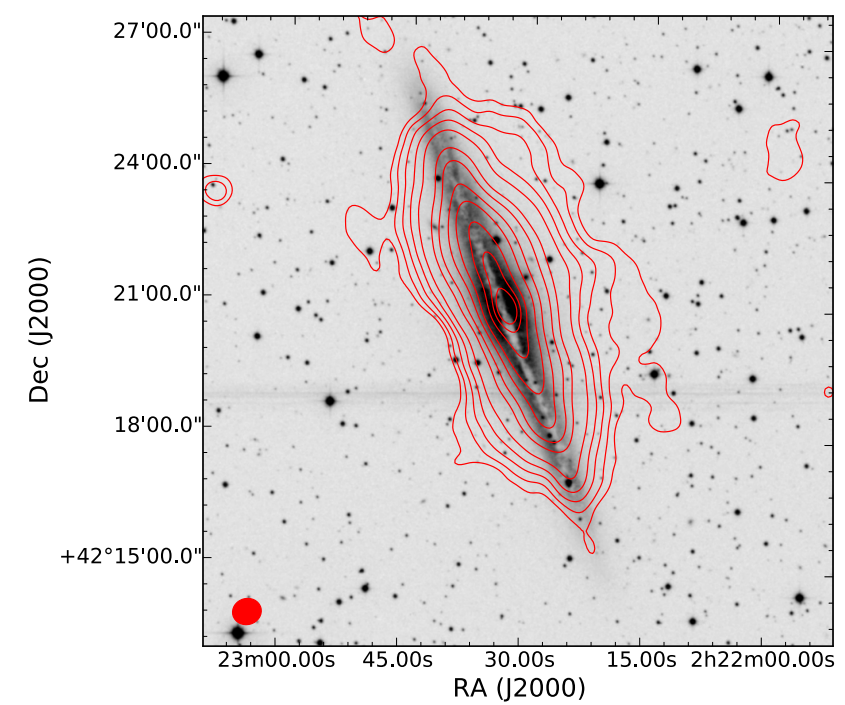

Fig. 4. LOFAR map of NGC 891 at a resolution of $40.0^{\prime \prime} \times 35.7^{\prime \prime}$ shown by the filled ellipse in the bottom left, overlaid onto an optical image from the Digitized Sky Survey. Contours are at 3, 5, 8, 12, 18, 32, $44,88,164,200 \times \sigma$ where $\sigma=1.1 \mathrm{mJy}^{-1}$ beam $^{-1}$ is the rms noise level.

in Fig. 4) is about $9 \mathrm{kpc}$ from the major axis. The extents are measured using the $3 \sigma$ level in Fig. 4.

The radio disk extends to about $16 \mathrm{kpc}$ from the centre along the major axis in the north and south. With the full extents of disk and the halo of about $32 \mathrm{kpc}$ along the plane and $14.5 \mathrm{kpc}$ perpendicular to the plane, respectively, the halo-to-disk extent ratio at $146 \mathrm{MHz}$ is $\approx 0.45$. This is similar to the ratio for NGC 253 at $200 \mathrm{MHz}$ (Kapińska et al. 2017). However, we caution that the halo-to-disk extent ratio is strongly dependent on sensitivity and angular resolution; a better way to quantify the halo emission is via the scale height (see Sect. 8).

Several features in the disk are resolved in the high-resolution image (Fig. 3, left panel). The most intense radio emission in the galaxy is seen in the central region of NGC 891 and the north of the disk due to the larger star-formation rate in this region of the disk. To the south of the disk, less intense radio emission is observed compared to the north.

The position of the radio supernova SN1986J is indicated by an arrow in Fig. 3 (left panel). SN1986J (van Gorkom et al. 1986) is one of the most luminous radio supernovas ever discovered (Bietenholz et al. 2010) and has been studied extensively since its discovery. The date of its explosion is uncertain; the best estimate is 1983.2 \pm 1.1 (Bietenholz et al. 2002). We detect SN1986J in our LOFAR high-resolution image (Fig. 3 left) as an unresolved point source located in the south-west of the disk. A Gaussian fit gives a flux density at $146 \mathrm{MHz}$ of $5.5 \pm 0.2 \mathrm{mJy} \mathrm{beam}^{-19}$ above the background disk emission at the position of $\operatorname{RA}(J 2000)=02^{\mathrm{h}} 22^{\mathrm{m}} 30^{\mathrm{s}} .8, \operatorname{Dec}(\mathrm{J} 2000)=+42^{\circ} 19^{\prime} 57^{\prime \prime}$.

\subsection{The $A M l$ images}

The images of NGC 891 at a central frequency of $15.5 \mathrm{GHz}$ observed with both the AMI arrays are shown in Fig. 5. For the SA image the final resolution is $142^{\prime \prime} \times 121^{\prime \prime}\left(6.5 \times 5.5 \mathrm{kpc}^{2}\right)$ and $36^{\prime \prime} \times 24^{\prime \prime}\left(1.6 \times 1.1 \mathrm{kpc}^{2}\right)$ for the LA image. For the $\mathrm{SA}$ and LA images the rms noise is $1.5 \mathrm{mJy} \mathrm{beam}^{-1}$ and $0.11 \mathrm{mJy}$ beam $^{-1}$, respectively.

\footnotetext{
9 The model by Bietenholz \& Bartel (2017) predicts a flux density of $4.8 \mathrm{mJy}$ for an age of $30.0 \mathrm{y}$ and frequency of $146.4 \mathrm{MHz}$, consistent with our observations.
}

The AMI SA image shows no distinct features for NGC 891 due to its low resolution. The AMI LA image is able to resolve the star-forming disk of NGC 891 and is similar to the image of the $\mathrm{H} \alpha$ line emission from ionized hydrogen gas (Fig. 6) and to the infrared image of thermal emission of warm dust at $24 \mu \mathrm{m}$ (see Fig. 6 in Whaley et al. 2009). The northern star-forming region stands also out in the emission of cold dust at $850 \mu \mathrm{m}$ (Alton et al. 1998), sub-millimetre line emission from rotational transitions of the $\mathrm{CO}$ molecule, a tracer for molecular hydrogen (Garcia-Burillo et al. 1992), and in optical H $\alpha$ line emission (e.g. Dahlem et al. 1994).

We measure an integrated flux density that is only about $2 \%$ lower for the LA image than for the SA image (avoiding the negative sidelobes), indicating that no significant flux density is lost in the LA image due to missing spacings. This seems surprising because the largest visible structure the LA is sensitive to is only about $3^{\prime}$. However, due to the highly elliptical shape of NGC 891 the visibilities at short baselines that are aligned at the position angle of the major axis can detect the entire flux density.

Very few previous observations of NGC 891 with a sufficiently small beam size to resolve the disk exist at similar frequencies. The closest in frequency are those of Gioia et al. (1982) and Dumke et al. (1995) who used the Effelsberg 100-m telescope at $10.7 \mathrm{GHz}$ and $10.55 \mathrm{GHz}$, respectively, and found integrated flux densities consistent with the AMI value (see Table 5).

The extent of the disk above the major axis is approximately $2 \mathrm{kpc}$ which is similar to the beam size at the distance of the galaxy, so that the disk thickness cannot be properly measured. No extended halo emission is observed at the given sensitivity. One feature, observed emanating from the region to the north of the galaxy (marked by "I"), extends to $\approx 3 \mathrm{kpc}$ from the plane and coincides with X-ray emission of hot gas observed with XMM-Newton at $0.4-0.75 \mathrm{keV}$ (Hodges-Kluck \& Bregman 2013). At 13-18 GHz, a substantial fraction of the emission we are observing is thermal, so this feature could be the result of outflow of warm gas (in addition to hot gas) from starforming regions in the northern region of the galaxy where the star-formation rate is larger (Dahlem et al. 1994).

\section{Comparison with other wavelengths}

\subsection{Radio continuum}

The most recent radio continuum data for comparison are the $1.5 \mathrm{GHz}$ and $6 \mathrm{GHz}$ VLA observations from the CHANG-ES survey (Wiegert et al. 2015; Schmidt 2016; Schmidt et al., in prep.). When the $146 \mathrm{MHz}$ image is smoothed to a larger beam (Fig. 4) in order to detect the most extended emission, the halo extends about as far out as in the $1.5 \mathrm{GHz} \mathrm{D}$-array image (with a similar beam size), but not further out. This is due to the relatively limited sensitivity of our LOFAR image. The exponential scale heights will give us a better indication of the halo extent, as will be shown in Sect. 8.

\section{2. $H \alpha$}

The diffuse $\mathrm{H} \alpha$ emission from the halo of NGC 891 has an exponential scale height of about $1 \mathrm{kpc}$ (Dettmar 1990). Rand et al. (1990) and Rossa et al. (2004) observed many vertical $\mathrm{H} \alpha$ filaments or "worms" extending up to $2 \mathrm{kpc}$ off the plane of the galaxy. They interpreted these "worms" as providing evidence for a galactic "chimney" mode (Norman \& Ikeuchi 1989). Rossa et al. (2004) speculated that the very narrow $\mathrm{H} \alpha$ filaments could be magnetically confined. 

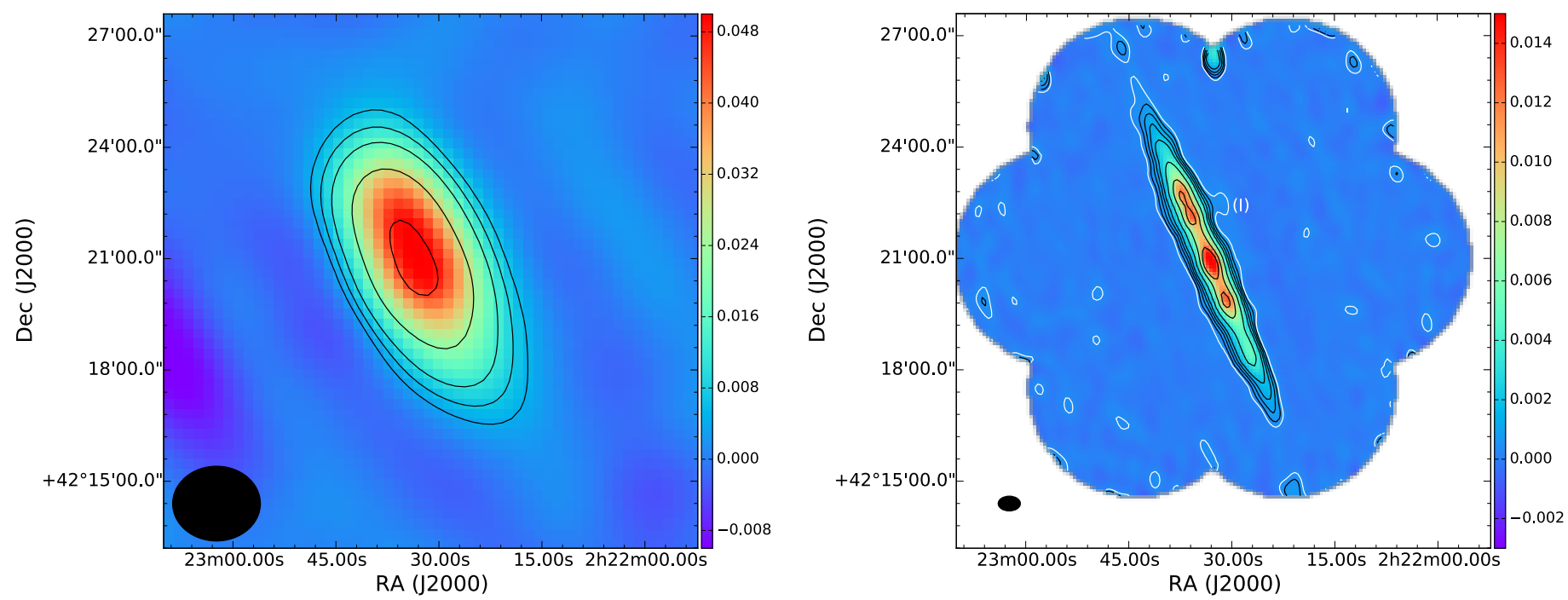

Fig. 5. AMI maps of NGC 891 observed at a central frequency of $15.5 \mathrm{GHz}$ with the Small Array (SA) at $142^{\prime \prime} \times 121^{\prime \prime}$ resolution $(l e f t)$ and the Large Array (LA) at $36^{\prime \prime} \times 24^{\prime \prime}$ resolution $(r i g h t)$. For the SA image, contours are at 3, 5, 8, 16, 32, $64 \times \sigma$ where $\sigma=1.5 \mathrm{mJy}^{-1}$ beam ${ }^{-1}$ is the rms noise level. For the LA image, contours are at 3 (white), 5, 8, 12, 18, 36, 72, $108 \times \sigma$ where $\sigma=0.11 \mathrm{mJy} \mathrm{beam}^{-1}$ is the rms noise level. The colour scales are in units of Jy/beam. The sizes of the synthesized beams are shown by the filled ellipses in the bottom left corners.

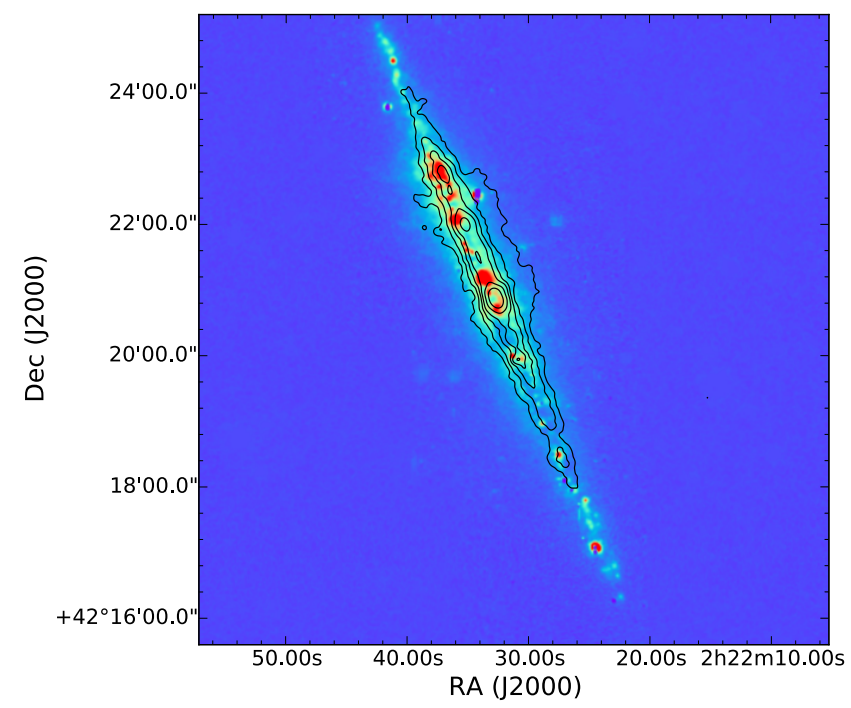

Fig. 6. H $\alpha$ image of NGC 891 from Rand et al. (1990) (in colours) with the high resolution LOFAR image (Fig. 3 left) overlaid as contours. Contours are at $12,18,25,32,44,64 \times \sigma$ where $\sigma=0.29$ mJy beam $^{-1}$ is the rms noise level.

We observe a relation between the high-resolution $146 \mathrm{MHz}$ radio continuum and $\mathrm{H} \alpha$ emission only in the $\mathrm{H} \alpha$ complex in the northern disk (Fig. 6). At this low frequency, we do not expect significant thermal emission (Sect. 6.1) while the $15.5 \mathrm{GHz}$ emission (Fig. 5 right) has a much larger thermal fraction (Sect. 6.2) and hence is more similar to the $\mathrm{H} \alpha$ image. However, the observations at $15.5 \mathrm{GHz}$ are not sensitive enough to detect diffuse thermal emission from the halo.

Figure 18 shows a composite of radio, $\mathrm{H} \alpha$, and optical emission.

\subsection{Neutral gas}

The galactic fountain model is invoked to explain the huge halo of neutral atomic Hi gas of NGC 891 (Oosterloo et al. 2007). The extent is up to $22 \mathrm{kpc}$ from the plane in the north-western quadrant. The exponential scale height increases from $1.25 \mathrm{kpc}$ in the central regions to about $2.5 \mathrm{kpc}$ in the outer parts beyond about $15 \mathrm{kpc}$ radius ("flaring"). The bulk of the cold CO-emitting molecular gas and the cold dust, on the other hand, are much more concentrated to the plane (Scoville et al. 1993; Alton et al. 1998), but some CO emission could be traced up to $1.4 \mathrm{kpc}$ height above the plane (Garcia-Burillo et al. 1992) and infrared emission up to $2.5 \mathrm{kpc}$ height (Whaley et al. 2009).

We do not observe such a large extension in the northwestern quadrant as seen in $\mathrm{H}_{\mathrm{I}}$ by Oosterloo et al. (2007). The size of the radio halo is limited by the synchrotron lifetime of the cosmic-ray electrons of about $2 \times 10^{8} \mathrm{yr}$ (Sect. 9.2), longer than the duty cycle of a typical galactic fountain of about $10^{8} \mathrm{yr}$ (Fraternali 2017). However, the north-western extension is much larger than a typical fountain because either its timescale is larger or the origin is different.

\subsection{X-rays}

X-ray observations performed by Bregman \& Pildis (1994) were able to detect a considerable amount of diffuse X-ray emission from the halo of NGC 891. The vertical profile is Gaussian with a vertical scale height of $3.5 \mathrm{kpc}$, corresponding to a full width at half maximum of $5.8 \mathrm{kpc}$ (Bregman \& Houck 1997). Temple et al. (2005) observed X-ray emission protruding from the disk in the north-western direction up to approximately $6 \mathrm{kpc}$ which showed a sharp cut-off, suggesting that this is the maximum extent that the outflowing hot gas has reached. The authors also concluded that NGC 891 has a larger star-formation rate than a normal spiral, but not as extreme as the starburst galaxy NGC 253. Cosmic rays and magnetic fields will also be transported by the outflow, but radio emission cannot be detected at such large heights due to energy losses of the cosmicray electrons and the limited sensitivity of present-day radio observations.

\section{NGC 891's spectral properties}

\subsection{Thermal and nonthermal emission}

To measure the nonthermal intensity $I_{\mathrm{n}}$ and the magnetic field strength, subtracting the free-free (thermal) emission is 

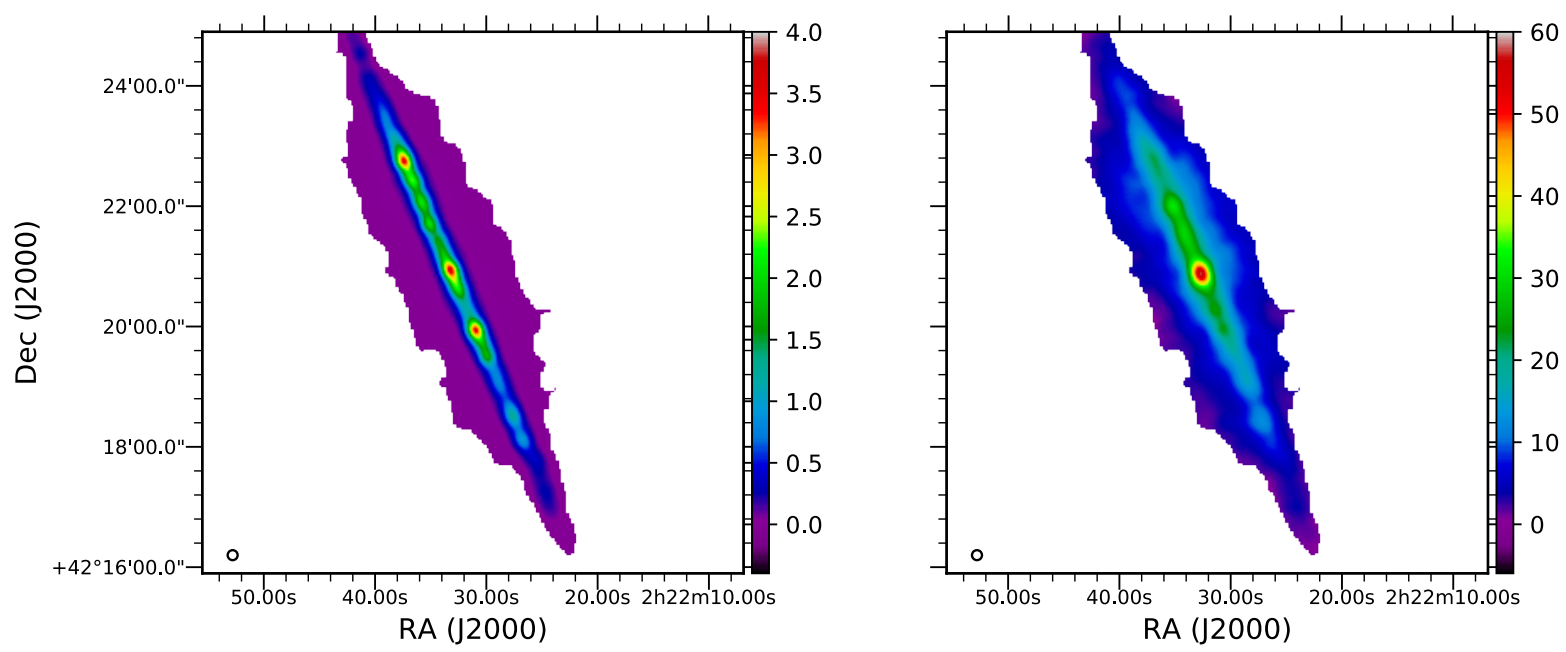

Fig. 7. Thermal map (left panel) and nonthermal map (right panel) of NGC 891 at $146 \mathrm{MHz}$ at a resolution of $12^{\prime \prime}$, shown by the circle in the bottom left corner. The colour scale is in $\mathrm{mJy}_{\text {beam }}{ }^{-1}$.

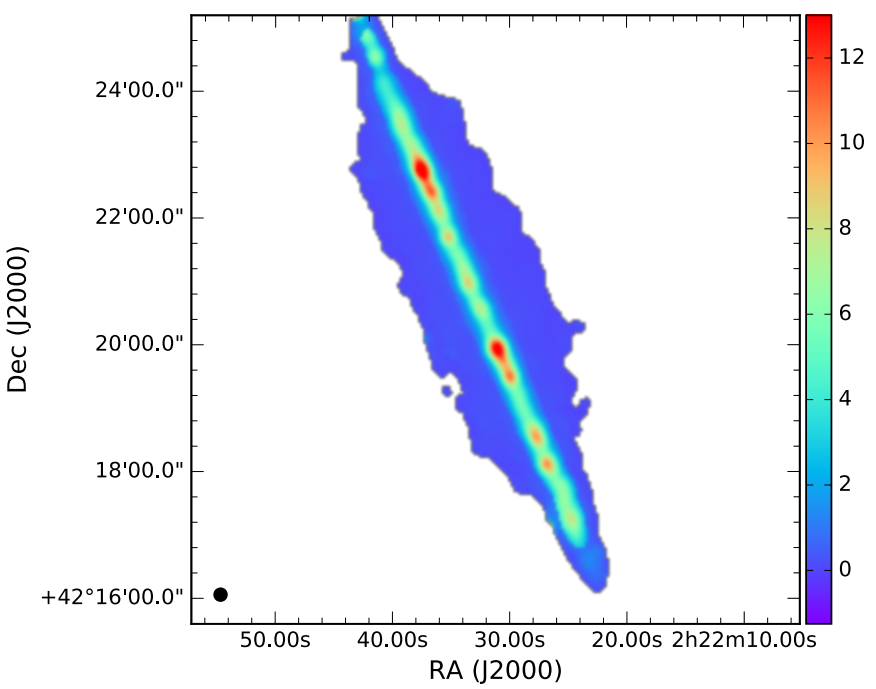

Fig. 8. Thermal fraction map of NGC 891 at $146 \mathrm{MHz}$ at a resolution of $12^{\prime \prime}$. The colour scale is in percent. The beam is shown in the bottom left corner.

essential. Furthermore, in order to investigate the energy losses of the CREs, the nonthermal spectral index $\alpha_{\mathrm{n}}\left(I_{\mathrm{n}} \propto v^{\alpha_{\mathrm{n}}}\right)$ needs to be known.

Our AMI LA image cannot be used as a tracer of thermal emission because the angular resolution is too coarse and the emission is still dominated by nonthermal emission (Sect. 6.2). A $1.5 \mathrm{GHz}$ thermal map at a resolution of $12^{\prime \prime}$ was derived from the CHANG-ES VLA data (Schmidt 2016, Schmidt et al. in prep.), based on an $\mathrm{H} \alpha$ image corrected for internal extinction with help of $24 \mu \mathrm{m}$ dust emission. In the inner disk of edge-on galaxies, the $24 \mu \mathrm{m}$ dust emission may become optically thick, so that the extinction correction may be insufficient and hence may lead to an underestimate of the thermal emission (Vargas et al. 2018). This effect was taken into account when estimating the thermal emission the $1.5 \mathrm{GHz}$, but leads to a large relative uncertainty of about $40 \%$.

The integrated thermal flux density of $53 \pm 23 \mathrm{mJy}$ corresponds to a thermal fraction of $7 \pm 3 \%$ at $1.5 \mathrm{GHz}$. This thermal map was scaled to $146 \mathrm{MHz}$, using the spectral index of optically thin thermal emission of -0.1 . If the assumption of optically thin emission is not valid at $146 \mathrm{MHz}$ in dense regions of the disk, the thermal emission will be overestimated. Our high resolution LOFAR image was convolved to the same resolution and re-gridded to the same grid size as the thermal map. The scaled thermal map was subtracted from the LOFAR image to produce the nonthermal image of NGC 891 at $146 \mathrm{MHz}$.

The thermal and nonthermal maps at $146 \mathrm{MHz}$ and at a resolution of $12^{\prime \prime}$ are shown in Fig. 7. The thermal image displays a thin disk plus a weak diffuse halo, similar to the maps of thermal emission of cold dust observed at $850 \mu \mathrm{m}$ (Alton et al. 1998; Israel et al. 1999) and of warm dust observed at $24 \mu \mathrm{m}$ (Whaley et al. 2009). In contrast to the thermal emission, the nonthermal disk is not "thin" and reveals a smooth transition to the halo.

The thermal fraction image at $146 \mathrm{MHz}$ was computed from the thermal image (Fig. 7 left) and a total intensity image at the same resolution of $12^{\prime \prime}$ and is shown in Fig. 8.

The thermal fractions in the disk are between $5 \%$ and $10 \%$ at $146 \mathrm{MHz}$ compared to $10-20 \%$ at $1.5 \mathrm{GHz}$. The largest thermal fraction of approximately $16 \%$ at $146 \mathrm{MHz}$ and $30 \%$ at $1.5 \mathrm{GHz}$ occurs in the northern disk of the galaxy. The halo reveals very small thermal fractions at $146 \mathrm{MHz}$ of $0.1-0.2 \%$ compared to $0.7-0.9 \%$ at $1.5 \mathrm{GHz}$, meaning that the radio emission observed in the halo is almost entirely nonthermal.

Free-free absorption by ionized gas in the disk lowers the radio synchrotron intensity at $146 \mathrm{MHz}$ (Sect. 6.4) and causes a further overestimation of the thermal fraction. Therefore, the thermal fractions at $146 \mathrm{MHz}$ estimated for the disk should be regarded as upper limits. Further observations at frequencies lower than $146 \mathrm{MHz}$ are needed to measure thermal absorption and correct the synchrotron intensity (see Sect. 9.1).

\subsection{Integrated spectrum of NGC 891}

We obtain an integrated flux density of NGC 891 with LOFAR at $146 \mathrm{MHz}$ of $2.85 \pm 0.28 \mathrm{Jy}$. The flux density of SN1986J was subtracted. The largest cause of uncertainty (about 10\%) is the limited accuracy of the beam model of LOFAR affecting the transfer of gains. The integrated flux density with AMI at $15.5 \mathrm{GHz}$ is $120 \pm 12 \mathrm{mJy}$, assuming a $10 \%$ uncertainty.

A whole range of flux density measurements from the literature was found with many of the flux density measurements taken from Hummel et al. (1991). Several flux density measurements from the literature were found to have 
either no uncertainty quoted or seriously underestimated their uncertainties by only including the rms noise (of typically a few \%) and not including any calibration uncertainty. In these cases we have inserted a $10 \%$ uncertainty to these flux density values. A full list of the flux density measurements is given in Table 5.

We subtracted the thermal emission at all frequencies, based on the value of $53 \pm 23 \mathrm{mJy}$ at $1.5 \mathrm{GHz}$ (Schmidt 2016; Schmidt et al., in prep.) and using the spectral index of optically thin thermal emission of -0.1 . The thermal fraction of the integrated flux density is $\leq 2 \%$ at $146 \mathrm{MHz}, 7 \pm 3 \%$ at $1.5 \mathrm{GHz}$, and $35 \pm 15 \%$ at $15.5 \mathrm{GHz}$. The spectrum of integrated nonthermal emission is plotted in Fig. 9.

The thermal fraction of NGC 891 at $1.5 \mathrm{GHz}$ fits well to the average thermal fractions at $1.4 \mathrm{GHz}$ obtained for the samples of spiral galaxies by Niklas et al. (1997; $8 \pm 1 \%$ ), Marvil et al. $(2015 ; 9 \pm 3 \%)$, and Tabatabaei et al. $(2017 ; 10 \pm 9 \%)^{10}$. This result indicates that the $24 \mu \mathrm{m}$ intensities of edge-on galaxies indeed needs to be corrected for extinction, as proposed by Vargas et al. (2018).

The shape of the spectrum of total radio continuum emission is determined by the relative contribution of the thermal freefree emission and the shape of the nonthermal (synchrotron) spectrum. As the disk dominates the radio emission from NGC 891, spectral effects in the disk are more important for the integrated emission than those in the halo. For example, at high frequencies, typically above $5 \mathrm{GHz}$, the increasing thermal fraction in the disk could lead to a spectral flattening. At lower frequencies, depending on the level of free-free absorption by ionized gas in the disk and the nature of the nonthermal spectrum, the total radio continuum spectrum could develop a spectral flattening, typically at $\$ 300 \mathrm{MHz}$. The shape of the nonthermal spectrum depends on the dominant energy loss/gain mechanisms which the synchrotron-emitting CREs undergo (see Sect. 9.2). A transition from dominating bremsstrahlung and adiabatic losses to dominating synchrotron and/or inverse-Compton (IC) losses leads to a spectral steepening by -0.5 beyond a certain frequency. Dominating ionization losses of low-energy CREs $(\lesssim 1.5 \mathrm{GeV})$ could also lead to a flattening of the nonthermal spectrum at low radio frequencies by +0.5 in regions of high gas density (e.g. Basu et al. 2015). We note that throughout the disk and halo spatially varying magnetic fields and gas densities (both neutral and ionized) lead to locally varying breaks in the CRE energy spectrum, such that the corresponding breaks in the galaxy's integrated radio continuum spectrum are smoothed out (Basu et al. 2015).

To study the nonthermal spectrum of NGC 891 we first model it using a simple power law of the form $S_{\mathrm{n}}(v)=a_{0} v^{\alpha_{\mathrm{n}}}$. Here, $S_{\mathrm{n}}$ is the nonthermal flux density, $\alpha_{\mathrm{n}}$ is the nonthermal spectral index and $a_{0}$ is the normalization at $1 \mathrm{GHz}$. We find the best fit $\alpha_{\mathrm{n}}=-0.78 \pm 0.02$, with a reduced $\chi^{2}=1.87$. The bestfit power-law spectrum is shown as the black dashed line in Fig. 9 (bottom panel). The expected total intensity spectrum after adding the thermal spectrum to the nonthermal power-law spectrum is shown as the black dashed line in the top panel of Fig. 9. This total flux density spectrum has a reduced $\chi^{2}=2.62$. Clearly, a simple power law does not represent the integrated spectrum well.

\footnotetext{
10 The average thermal fraction derived for a sample of star-forming galaxies by Klein et al. (2018) is more than twice larger, but these galaxies show indications for a break or an exponential decline in their nonthermal radio spectra which makes the estimate of the thermal fraction difficult.
}

Table 5. Integrated flux densities of NGC 891.

\begin{tabular}{cll}
\hline \hline$v(\mathrm{GHz})$ & Flux density (Jy) & Ref. \\
\hline 15.5 & $0.120 \pm 0.012$ & This work \\
10.7 & $0.152 \pm 0.026$ & Gioia et al. (1982) \\
10.7 & $0.155 \pm 0.010$ & Israel \& van der Hulst (1983) \\
10.55 & $0.183 \pm 0.010$ & Dumke et al. (1995) \\
8.7 & $0.171 \pm 0.023$ & Beck et al. (1979) \\
6.0 & $0.25 \pm 0.03$ & Schmidt et al. (in prep.) \\
4.995 & $0.29 \pm \mathbf{0 . 0 3}$ & Allen et al. (1978) \\
4.85 & $0.25 \pm \mathbf{0 . 0 3}$ & Gregory \& Condon (1991) \\
4.8 & $0.29 \pm \mathbf{0 . 0 3}$ & Stil et al. (2009) \\
4.75 & $0.30 \pm \mathbf{0 . 0 3}$ & Gioia et al. (1982) \\
2.695 & $0.43 \pm 0.06$ & Kazès et al. (1970) \\
2.695 & $0.38 \pm 0.03$ & de Jong (1967) \\
1.5 & $0.74 \pm 0.04$ & Schmidt et al. (in prep.) \\
1.49 & $0.74 \pm 0.02$ & Hummel et al. (1991) \\
1.49 & $0.66 \pm 0.06$ & Gioia \& Fabbiano (1987) \\
1.49 & $0.70 \pm \mathbf{0 . 0 7}$ & Condon (1987) \\
1.412 & $0.77 \pm \mathbf{0 . 0 8}$ & Allen et al. (1978) \\
0.75 & $1.4 \pm \mathbf{0 . 1 4}$ & Heeschen \& Wade (1964) \\
0.61 & $1.53 \pm 0.08$ & Hummel et al. (1991) \\
0.61 & $1.6 \pm \mathbf{0 . 1 6}$ & Allen et al. (1978) \\
0.408 & $1.8 \pm 0.1$ & Gioia \& Gregorini (1980) \\
0.408 & $1.7 \pm \mathbf{0 . 2}$ & Baldwin \& Pooley (1973) \\
0.327 & $2.1 \pm \mathbf{0 . 2}$ & Hummel et al. (1991) \\
0.330 & $2.1 \pm 0.1$ & Rengelink et al. (1997) \\
0.146 & $2.85 \pm 0.28$ & This work \\
0.0575 & $6.6 \pm 1.8$ & Israel \& Mahoney (1990) \\
\hline & &
\end{tabular}

Notes: Uncertainties marked in bold were increased from their original values, as explained in the text.

In order to assess any curvature in the nonthermal spectrum of NGC 891, we empirically model it with a second-order polynomial of the form $\log S_{\mathrm{n}}(v)=\log a_{0}+\alpha \log v+\beta(\log v)^{2}$. Here, $\alpha$ is the spectral index and $\beta$ is the curvature parameter. The best fit values are found to be $a_{0}=0.94 \pm 0.02, \alpha=$ $-0.76 \pm 0.02$, and $\beta=-0.14 \pm 0.03$. The best-fit model is shown as the green solid line in Fig. 9. The reduced $\chi^{2}$ for the fit is 0.86 and that for the total emission is 1.32 , suggesting that the nonthermal spectrum significantly deviates from that of a simple power law.

To understand the physical origin of the curvature, we also performed modelling of the nonthermal spectrum of NGC 891 with a spectral break given by $S_{\mathrm{n}}(v)=a_{0} v^{\alpha_{\mathrm{inj}}} /\left[\left(v / \nu_{\mathrm{br}}\right)^{0.5}+1\right]$. Here, $\alpha_{\text {inj }}$ is the injection spectral index of the CREs and $v_{\mathrm{br}}$ is the break frequency beyond which the spectrum steepens by -0.5 . Unfortunately, with the current data the parameters for this model cannot be well constrained. We therefore fixed $\alpha_{\text {inj }}=-0.6$ (see Fig. 3 in Caprioli 2011). This yields a break at $v_{\mathrm{br}}=3.7 \pm 1.3 \mathrm{GHz}$ which corresponds to a synchrotron age (Eq. (3)) of $\lesssim 2.5 \times 10^{7} \mathrm{yr}$ for the CREs in the disk emitting in a magnetic field of $\gtrsim 10 \mu \mathrm{G}$ (Sect. 7). The best fit is shown as the blue dashed-dotted line in Fig. 9. It gives a reduced $\chi^{2}=1.19$ for the nonthermal emission and a reduced $\chi^{2}=$ 1.57 for the total emission, better than the values for a simple power law.

As discussed above, the curvature in the nonthermal spectrum could also arise as the result of synchrotron-free absorption and/or ionization losses at frequencies below about $150 \mathrm{MHz}$ (see Fig. 3 in Basu et al. 2015). To investigate the first scenario, a rigorous modelling of the synchrotron radiative transfer in an 

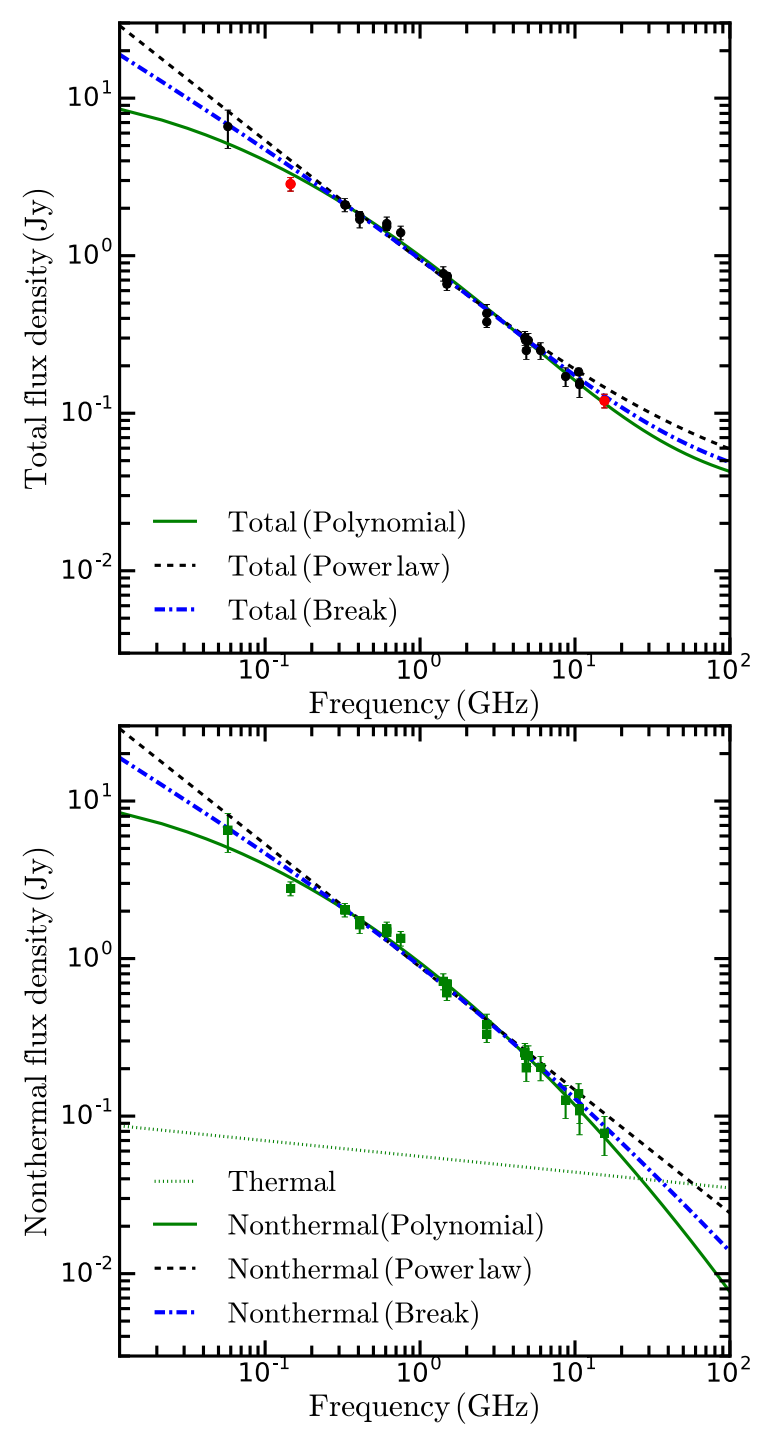

Fig. 9. Top: integrated total flux densities of NGC 891 as listed in Table 5. The two new total flux densities from this paper are marked in red. Bottom: integrated nonthermal flux densities of NGC 891 after subtracting an estimate of the optically thin thermal free-free emission (shown as the green dotted line) from the total flux densities. The black dashed, solid green, and blue dashed-dotted lines in the bottom panel show the best-fit power law, second-order polynomial model, and spectral break model to the nonthermal emission, respectively. The corresponding lines in the top panel show the expected total flux density spectrum after adding the thermal free-free emission to the different models of the nonthermal spectrum.

inclined disk is necessary, while for the later case additional information on the $\gamma$-ray spectrum is needed. Given the scarcity of low-frequency observations and the large uncertainty in the estimated thermal emission, the current data are insufficient to constrain the origin of the curvature in the nonthermal spectrum of NGC 891.

To better constrain the radio continuum spectrum, additional radio frequency observations at low frequencies would be helpful. The $146 \mathrm{MHz}$ flux density derived in this paper seems to indicate a spectral flattening at low frequencies by freefree absorption. However, this is inconsistent with the value at $57.5 \mathrm{MHz}$. The latter value is based on observations with the Clark Lake Radio Observatory synthesis radio telescope with a synthesized beam size of about 7' (Israel \& Mahoney 1990). As the frequency range below $100 \mathrm{MHz}$ is crucial to detect spectral flattening by free-free absorption, new observations with higher resolution are needed, e.g. with the LOFAR Low Band Antenna (see Sect. 9.1).

\subsection{Spectral index map of NGC 891}

To derive spectral index maps of high accuracy, the frequency span should be as large as possible. However, our new AMI image at $15.5 \mathrm{GHz}$ does not provide sufficient angular resolution to be combined with our new LOFAR image at $146 \mathrm{MHz}$. Hence, spectral index maps were created from the CHANG-ES VLA image of NGC 891 at $1.5 \mathrm{GHz}$ (Schmidt 2016; Schmidt et al., in prep.) ${ }^{11}$ and our LOFAR image. In order to be sensitive to the same angular scales, both images were generated with the same minimum and maximum $u v$ distance and the same weighting scheme, smoothed to two different resolutions, $10^{\prime \prime}$ and $20^{\prime \prime}$, and placed onto the same grid via the AIPS task OHGEO. Only pixels with flux densities above $8 \times$ the $\mathrm{rms}(\sigma)$ level in the four input images were used.

The minimum projected baseline for the VLA is about $27 \mathrm{~m}$, corresponding in units of wavelength to a baseline about $270 \mathrm{~m}$ for LOFAR at $146 \mathrm{MHz}$. This $u v_{\min }$ value corresponds to an angular scale of about $25^{\prime}$ which is much larger than the angular scale of NGC 891. Hence, we do not expect the spectral index distribution to be affected by systematic errors due to missing angular scales.

Before determining the spectral index, both images were set on the same flux density scale. The $146 \mathrm{MHz}$ LOFAR image was calibrated on the Scaife \& Heald flux density scale (Scaife \& Heald 2012), while the $1.5 \mathrm{GHz}$ image was calibrated on the Perley \& Butler (2013) flux density scale. We first converted the 1.5 GHz image to the Baars scale (Baars et al. 1977) by a factor of 1.021 taken from Table 13 of Perley \& Butler (2013). We then converted this flux density to the KPW scale (Kellermann et al. 1969) which is identical to the Scaife \& Heald flux density scale at frequencies above $325 \mathrm{MHz}$, using a factor of 1.029 taken from Table 7 of Baars et al. (1977).

The spectral index between $146 \mathrm{MHz}$ and $1.5 \mathrm{GHz}$ was computed pixel by pixel and is shown in Fig. 10 along with the image of uncertainties (errors) due to rms noise for both resolutions. The spectral index map at $10^{\prime \prime}$ resolution (Fig. 10 top) reveals great detail on the disk's spectral features. We observe very flat spectra in the central region $(\alpha \approx-0.3)$, coincident with prominent $\mathrm{H}_{\text {II }}$ regions. In other regions in the disk we observe spectra with $\alpha \approx-0.5$. This is flatter than what is observed at higher frequencies. Schmidt et al. (in prep.) found the spectral index between $1.5 \mathrm{GHz}$ and $6 \mathrm{GHz}$ to be $\alpha \approx-0.7$ in the disk. Immediately away from the disk, we observe spectral indices of $\approx-0.6$ to -0.7 .

The spectral index map at $20^{\prime \prime}$ resolution (Fig. 10 bottom) shows a similar steepening of the spectral index from the disk to the halo. Due to the higher sensitivity with respect to weak extended emission, we can trace the spectral index further away from the disk into the halo.

\subsection{Nonthermal spectral index of NGC 891}

The spectral index is contaminated by thermal emission that is unrelated to CREs and magnetic fields. Therefore, we computed a map of the nonthermal spectral index $\alpha_{\mathrm{n}}$, using the nonthermal maps at $146 \mathrm{MHz}$ at $12^{\prime \prime}$ resolution (Fig. 7 right) and at $1.5 \mathrm{GHz}$ at the same resolution.

${ }_{11}$ We did not use the $6 \mathrm{GHz}$ CHANG-ES image because the signal-tonoise ratios are smaller than those at $1.5 \mathrm{GHz}$. 
D. D. Mulcahy et al. : Cosmic rays and magnetic fields in the halo of NGC 891
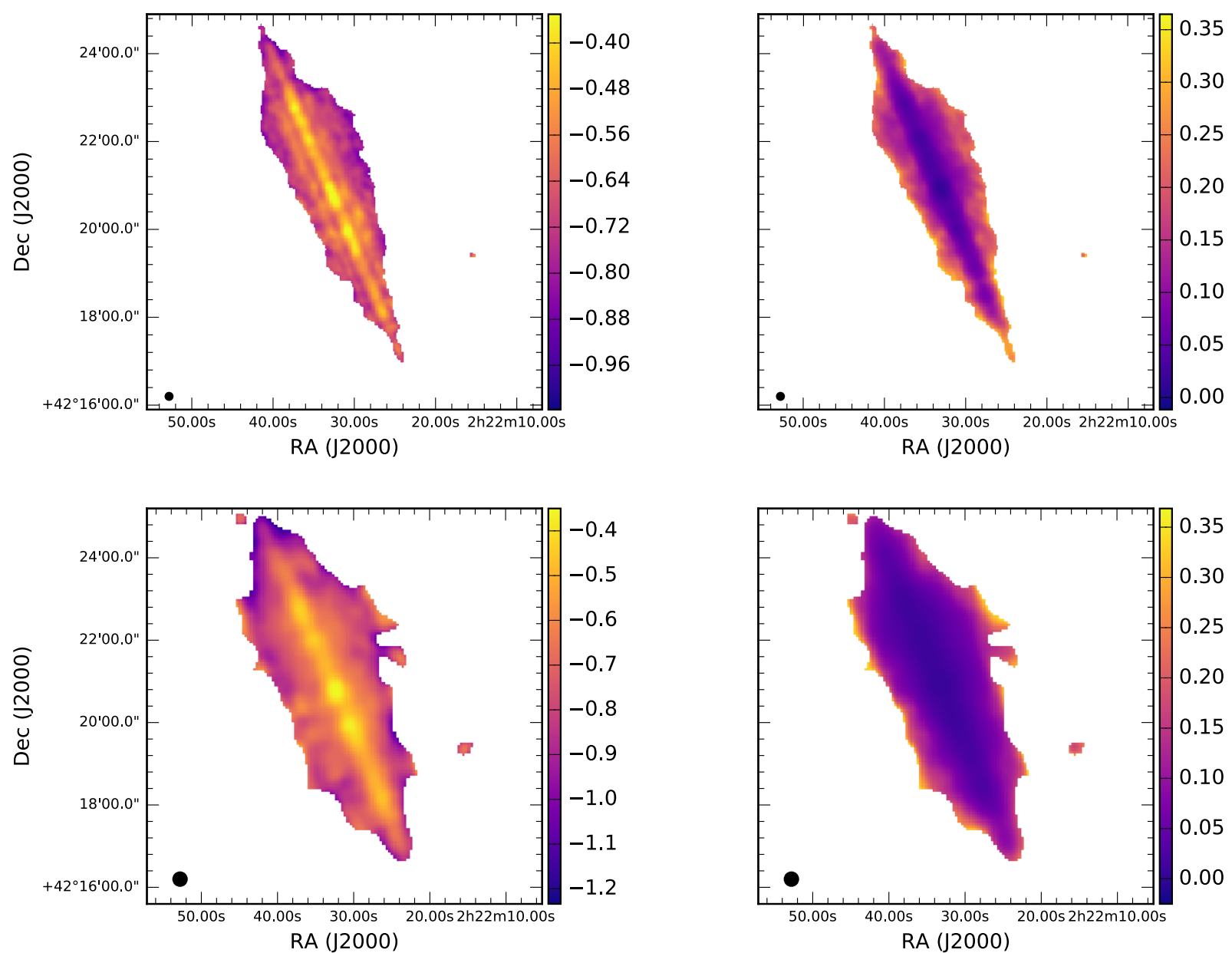

Fig. 10. Spectral index (left panel) and error map (right panel) of NGC 891 between $146 \mathrm{MHz}$ and $1.5 \mathrm{GHz}$ at resolutions of $10^{\prime \prime}$ (top panel) and $20^{\prime \prime}$ (bottom).
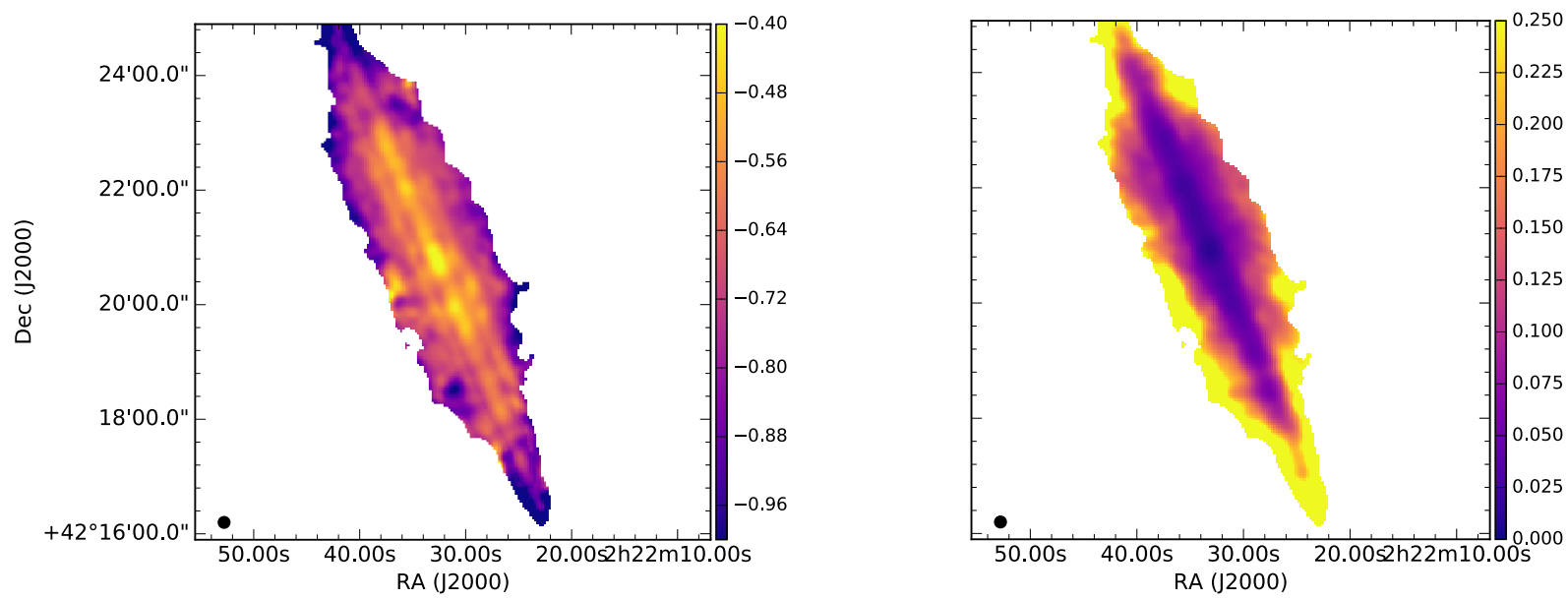

Fig. 11. Maps of nonthermal spectral index (left panel) and corresponding error map (right panel) between $146 \mathrm{MHz}$ and $1.5 \mathrm{GHz}$ at $12^{\prime \prime}$ resolution. The beam is shown in the bottom left corner.

The nonthermal spectral indices in the disk of the galaxy show significant signs of flattening. In the very centre of the galaxy we measure a spectral index of -0.37 . We measure spectral indices of -0.43 to -0.48 in the northern and southern star-forming regions of the disk, respectively. Other regions in the disk show spectral indices of -0.5 to -0.6 .

In the disk the observed CRE population is a superposition of various spectral ages with young CREs located in the major starforming regions, while older CREs would exist in the inter-arm regions (Tabatabaei et al. 2007) and in the halo. Therefore, we expect to observe the injection spectral index in star-forming regions and a steeper spectral index in inter-arm regions and in the halo. Indeed, a significant arm-interarm contrast was observed for M51 (Mulcahy et al. 2014), with $\alpha_{\mathrm{n}}=-0.8$ between $151 \mathrm{MHz}$ and $1.4 \mathrm{GHz}$ in the inter-arm regions.

CREs are accelerated in the shock fronts of supernova remnants (SNRs). Observations of the $\gamma$-ray emission from bright SNRs yield an average energy spectral index of CRs in the 


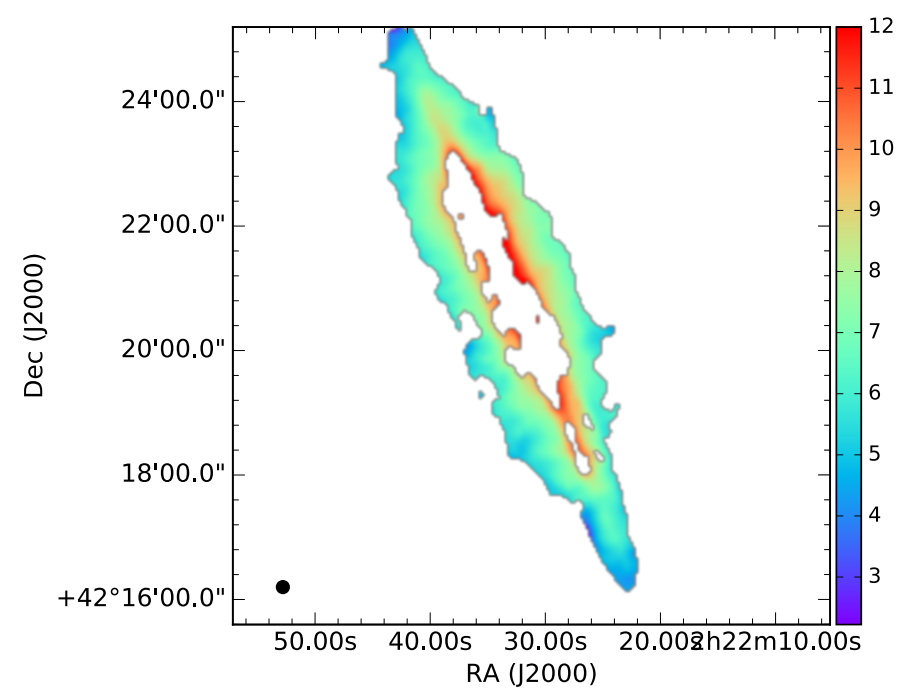

Fig. 12. Equipartition strength of the magnetic field $B$ at 12 " resolution, in units of $\mu \mathrm{G}$, determined by assuming energy equipartition between cosmic rays and magnetic fields. Pixels with $\alpha_{\mathrm{n}}>-0.6$ are blanked. The beam is shown in the bottom left corner.

energy range $100 \mathrm{MeV}-50 \mathrm{GeV}$ of $\gamma_{\mathrm{inj}}-2.2 \pm 0.2$ (Caprioli 2011) where the CRE number density spectrum is a power law as a function of energy, $N \propto E^{\gamma_{\text {inj }}}$. This is equivalent to an average injection spectral index in the radio range of $\alpha_{\text {inj }}-0.6 \pm 0.1$, consistent with the average spectral index of the radio emission from SNRs of -0.5 , though with a large dispersion (Green 2014).

Nonthermal spectral indices of $\alpha_{\mathrm{n}} \approx-0.7$ between $1.4 \mathrm{GHz}$ and $4.86 \mathrm{GHz}$ are observed in the disk of NGC 891 (Heesen et al. 2018a). CREs in the disk emitting at frequencies of a few $\mathrm{GHz}$ are still young and have almost maintained their injection spectrum. At lower frequencies we are observing flatter spectra with $\alpha_{\mathrm{n}, 146 \mathrm{MHz}-1.5 \mathrm{GHz}}>\alpha_{\text {inj }}$ which indicates that we are observing significant thermal (free-free) absorption of synchrotron emission in star-forming regions in the disk (see Sect. 9.1).

In the halo we find $\alpha_{\mathrm{n}} \leq-0.8$, revealing CRE ageing through synchrotron and/or IC emission. These spectra are still significantly flatter compared to what is observed at higher frequencies (Heesen et al. 2018a). The nonthermal spectra in the halo steepen with increasing frequency, as expected from energy losses of CREs by synchrotron or IC emission (see Sect. 9.2).

\section{Magnetic field strength in the halo of NGC 891}

The strength of the magnetic field (including turbulent and ordered components) can be determined from the nonthermal emission by assuming equipartition between the energy densities of total cosmic rays and magnetic fields, using the revised formula of Beck \& Krause (2005). The equipartition magnetic field strength $B$ scales with the synchrotron intensity $I_{\text {syn }}$ as:

$B \propto\left(\frac{I_{\text {syn }}}{(K+1) L}\right)^{1 /\left(3-\alpha_{\mathrm{n}}\right)}$

where $\alpha_{\mathrm{n}}$ is the nonthermal spectral index. The adopted ratio of CR proton to electron number densities of $K=100$ is a reasonable assumption in the star-forming regions in the disk (Bell 1978). Strong energy losses of CREs due to synchrotron and/or IC emission (Sect. 9.2) are signified by $\alpha_{\mathrm{n}} \leq-1.1$ in Fig. 11 and occur at the outskirts of NGC 891. These lead to an increase of $K$ by a factor of a few and hence to an underestimate of $B$ in these regions by about $10-30 \%$.

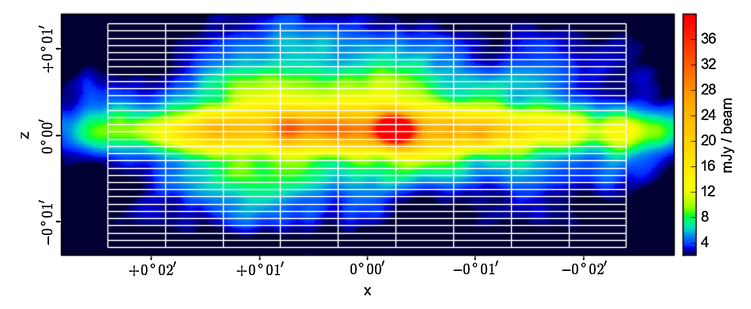

Fig. 13. Image of NGC 891 with indication of the boxes used for computing the average intensities, at different distances from the major axis of the galaxy's projected disk, and in 9 different strips along the major axis.

The effective path length through NGC 891, $L$, is assumed to be 0.8 times the radio diameter, resulting in $L=19 \mathrm{kpc}$. Depending on the shape and extent of the magnetic fields in the halo, $L$ decreases with distance from the major axis and also with distance from the galaxy's centre along the major axis. We assumed a constant value of $L$ which results in an underestimate of $B$ by another $10-30 \%$. An improved model of the distribution of magnetic field strengths will be presented in a forthcoming paper (Schmidt et al. in prep.).

We created an image of the equipartition field strength $B$ in NGC 891 from the nonthermal total intensity (Fig. 7 right) and the nonthermal spectral index (Fig. 11 left), presented in Fig. 12. We blanked pixels with $\alpha_{\mathrm{n}}>-0.6$ as such regions are likely suffering from free-free absorption and would significantly underestimate the magnetic field strength. Blanking regions where $\alpha_{\mathrm{n}}>-0.6$ results in a large fraction of the inner disk without a magnetic field strength estimate and highlights the difficulty posed by free-free absorption in determining the magnetic field strength.

The field strength is found to be highest near to the plane with a field strength of $10-11 \mu \mathrm{G}$, similar to the average field strength in the disks of mildly inclined galaxies (Beck 2015) and of edgeon galaxies (Krause et al. 2018). The average equipartition field strength in the halo, between $1 \mathrm{kpc}$ and $3 \mathrm{kpc}$ height, is $7 \mu \mathrm{G}$ with a standard deviation of $2 \mu \mathrm{G}$.

\section{Radio emission profiles and scale heights}

The vertical distributions of radio emission and their scale heights in the halos of star-forming edge-on galaxies have been studied in numerous works (e.g. Dumke et al. 1995; Heesen et al. 2009; Krause et al. 2018) and is a vital tool for the analysis of cosmic ray propagation and CRE energy losses. Data below $300 \mathrm{MHz}$ have never been used before, because sensitivity and angular resolution at low frequencies were insufficient so far. The MWA images of NGC 253 between 88 and $215 \mathrm{MHz}$ have too low angular resolutions (between $5^{\prime}$ and $2^{\prime}$ ) for this purpose.

The sensitivity of the observations limits the detectability of an extended halo. Therefore, the total observed extent is not suited as a physical parameter describing the halo emission, and a more objective parameter such as the scale height is required (Krause et al. 2018).

To allow for testing CRE propagation models (see Sect. 9.3), we need to measure the scale heights of the nonthermal emission at $146 \mathrm{MHz}$ and $1.5 \mathrm{GHz}$. However, free-free absorption in the disk hampers a reliable estimate of thermal and nonthermal emission at $146 \mathrm{MHz}$, so that we use instead the total emission at this frequency. The difference between these two quantities can be neglected because the thermal fractions are small (Sect. 6.1). At $1.5 \mathrm{GHz}$ we use the nonthermal map from Schmidt et al. (in prep.). 
D. D. Mulcahy et al. : Cosmic rays and magnetic fields in the halo of NGC 891

Table 6. Scale heights $h$ of the fits to the total intensity at $146 \mathrm{MHz}$ at $12^{\prime \prime}(0.55 \mathrm{kpc})$ resolution for the 9 strips at different distances, $x$, from the galaxy's centre along the major axis, and to the nonthermal intensity at $1.5 \mathrm{GHz}$, taken from Schmidt et al. (in prep.).

\begin{tabular}{ccccc|ccc|cc}
\hline \hline$x\left({ }^{\prime \prime}\right)$ & $x(\mathrm{kpc})$ & $\begin{array}{c}h_{\text {disk }}(\mathrm{kpc}) \\
146 \mathrm{MHz}\end{array}$ & $\begin{array}{c}h_{\text {halo }}(\mathrm{kpc}) \\
146 \mathrm{MHz}\end{array}$ & $\chi_{\text {red }}^{2}$ & $\begin{array}{c}h_{\text {disk }}(\mathrm{kpc}) \\
1.5 \mathrm{GHz}\end{array}$ & $\begin{array}{c}h_{\text {halo }}(\mathrm{kpc}) \\
1.5 \mathrm{GHz}\end{array}$ & $\chi_{\text {red }}^{2}$ & $q_{\text {disk }}$ & $q_{\text {halo }}$ \\
\hline+160 & +7.37 & $0.47 \pm 0.11$ & $3.6 \pm 2.0$ & 2.5 & $0.25 \pm 0.03$ & $1.66 \pm 0.07$ & 0.31 & $1.9 \pm 0.5$ & $2.1 \pm 1.2$ \\
+120 & +5.53 & $0.23 \pm 0.04$ & $2.0 \pm 0.2$ & 2.4 & $0.15 \pm 0.01$ & $1.40 \pm 0.03$ & 0.37 & $1.5 \pm 0.3$ & $1.5 \pm 0.2$ \\
+80 & +3.68 & $0.21 \pm 0.07$ & $2.0 \pm 0.2$ & 4.4 & $0.14 \pm 0.03$ & $1.38 \pm 0.06$ & 4.8 & $1.5 \pm 0.6$ & $1.4 \pm 0.2$ \\
+40 & +1.84 & $0.34 \pm 0.09$ & $2.1 \pm 0.4$ & 6.1 & $0.29 \pm 0.06$ & $1.48 \pm 0.19$ & 13 & $1.2 \pm 0.4$ & $1.4 \pm 0.3$ \\
0 & 0.00 & $0.26 \pm 0.06$ & $1.8 \pm 0.3$ & 12 & $0.13 \pm 0.02$ & $1.13 \pm 0.09$ & 30 & $2.0 \pm 0.6$ & $1.6 \pm 0.3$ \\
-40 & -1.84 & $0.40 \pm 0.19$ & $1.6 \pm 0.6$ & 9.0 & $0.21 \pm 0.06$ & $1.07 \pm 0.08$ & 7.0 & $1.9 \pm 1.1$ & $1.5 \pm 0.6$ \\
-80 & -3.68 & $0.30 \pm 0.06$ & $1.7 \pm 0.2$ & 2.8 & $0.21 \pm 0.04$ & $1.14 \pm 0.08$ & 4.6 & $1.4 \pm 0.4$ & $1.5 \pm 0.2$ \\
-120 & -5.53 & $0.30 \pm 0.06$ & $2.5 \pm 0.4$ & 1.3 & $0.22 \pm 0.03$ & $1.45 \pm 0.06$ & 1.1 & $1.4 \pm 0.3$ & $1.7 \pm 0.3$ \\
-160 & -7.37 & $0.39 \pm 0.08$ & $3.5 \pm 1.2$ & 3.9 & $0.21 \pm 0.02$ & $1.51 \pm 0.05$ & 0.26 & $1.9 \pm 0.4$ & $2.3 \pm 0.8$ \\
\hline
\end{tabular}

Notes: $x$ is positive on the north-eastern side and negative on the south-western side. $q$ is the ratio of the scale heights at $146 \mathrm{MHz}$ and $1.5 \mathrm{GHz}$.

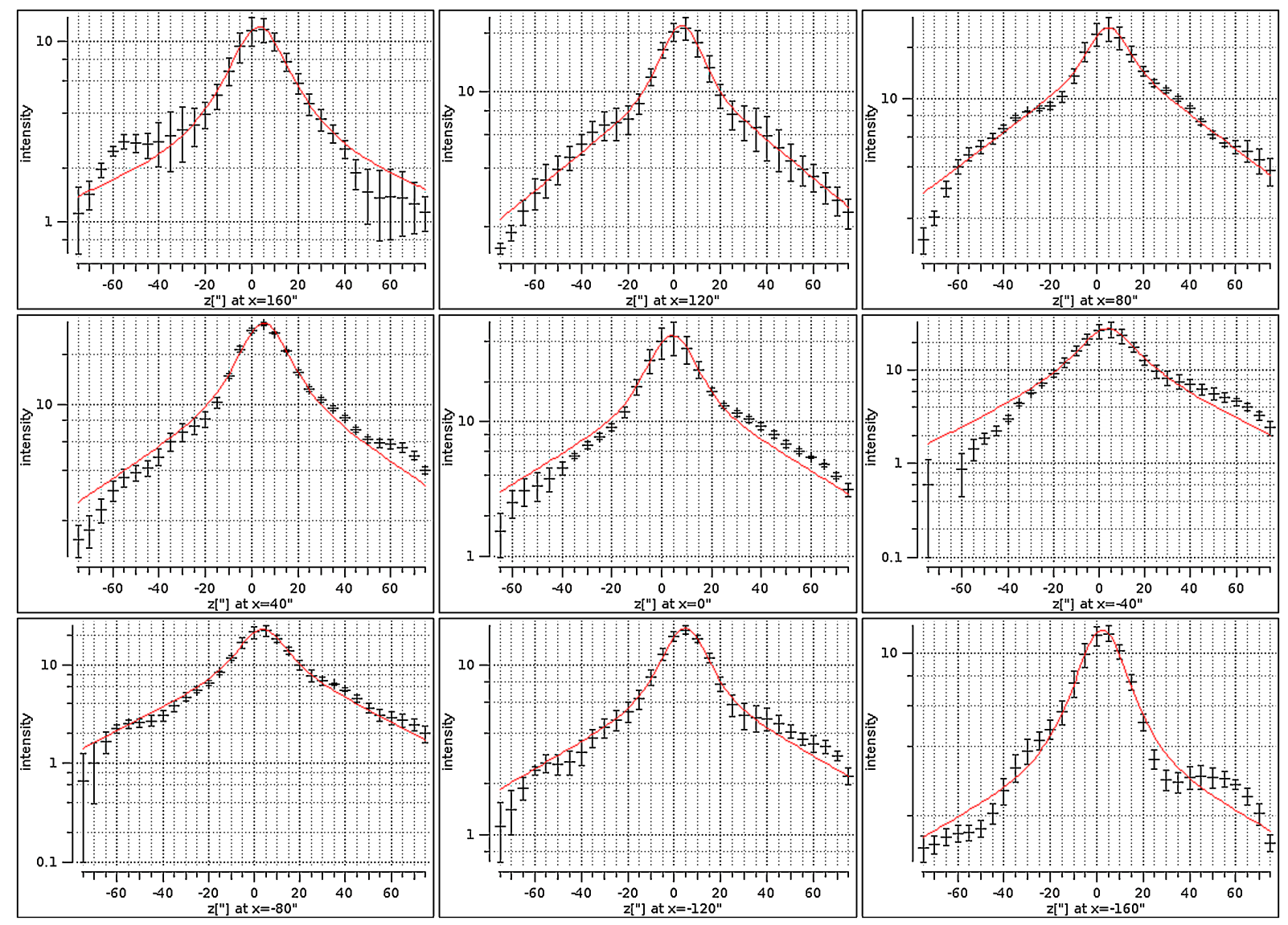

Fig. 14. Profiles of the average intensity at $146 \mathrm{MHz}$ at $12^{\prime \prime}$ resolution (in mJy beam ${ }^{-} 1$ ) as a function of distance $z$ from the major axis (in arcseconds, $10^{\prime \prime} \simeq 0.46 \mathrm{kpc}$ ), for the 9 strips of NGC 891 at different distances, $x$, from the galaxy's centre along the major axis, corresponding to Fig. 13. The first panel is at $\mathrm{x}=+7.37 \mathrm{kpc}$ (northern side), the last one at $x=-7.37 \mathrm{kpc}$ (southern side). $z$ is negative (positive) on the eastern (western) side of the halo. The red lines show the two-component exponential functions fitted to the data.

The disk scale heights of the nonthermal emission at $1.5 \mathrm{GHz}$ are on average $12 \%$ larger than those of the total emission, indicating that the nonthermal disk is broader than the thermal disk. The halo scale heights of the nonthermal and total emission at $1.5 \mathrm{GHz}$ are the same within the errors.

Following a similar approach as in Krause et al. (2018), we fitted two intrinsically exponential distributions to the vertical profiles of the disk and the halo. The vertical profile of the observed radio emission in an edge-on galaxy is a superposition of the vertical profile of the disk + halo emission, the radial profile of the disk emission projected onto the sky plane, and broadening by the telescope beam (see Müller et al. (2017) and Krause et al. (2018) for full details). For an almost perfectly edge-on galaxy such as NGC 891, the radial profile of the disk emission does not contribute to the vertical profile.

To obtain the profiles of radio emission at $146 \mathrm{MHz}$ with distance from the major axis for both the total intensity map and the nonthermal map (Fig. 7 right) at $12^{\prime \prime}$ resolution, we performed averaging within strips of $40^{\prime \prime}(\simeq 1.84 \mathrm{kpc})$ width and $75^{\prime \prime}$ $(\simeq 3.45 \mathrm{kpc})$ maximum height above and below the major axis, 


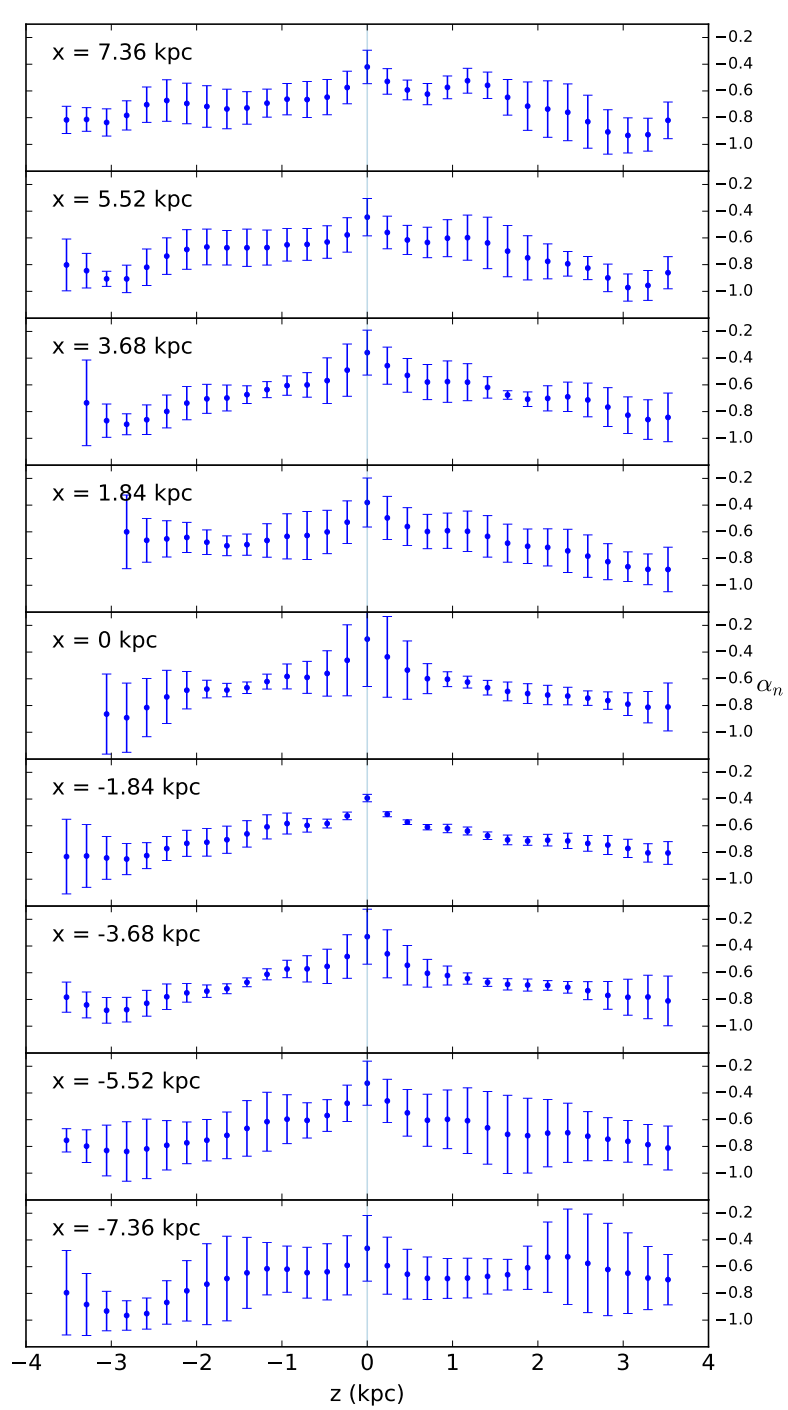

Fig. 15. Profiles of the nonthermal spectral index, $\alpha_{n}$, as a function of height $z$ above the major axis (in kpc) for the 9 strips at different distances, $x$, from the galaxy's centre along the major axis. The top four panels $(x>0)$ correspond to the northern side of the galaxy and the bottom four panels $(x<0)$ to the southern side; the middle panel is centred on the minor axis. $z$ is negative (positive) on the eastern (western) side of the halo.

applying the method described in Müller et al. (2017). Each data point is separated by $5^{\prime \prime}$, slightly less than half the FWHM of the beam. We also calculated profiles with different maximum heights above and below the major axis, without significantly different results. The profiles of the total intensity are presented in Fig. 14; those of the nonthermal intensity are very similar and are not shown. A visualisation of the distribution of strips is shown Fig. 13. The same process was also performed on the VLA $1.5 \mathrm{GHz}$ total intensity map from Schmidt et al. (in prep.). The distributions were fitted by a least-squares routine and are shown as red lines in Fig. 14. The corresponding scale heights, corrected for beam smearing, are summarized in Table 6 .

The exponential scale heights of the total radio emission at $146 \mathrm{MHz}$ for both the disk and halo are consistently larger than those at $1.5 \mathrm{GHz}$. The mean scale height of the disk emission is $0.32 \pm 0.08 \mathrm{kpc}$ at $146 \mathrm{MHz}$, compared to $0.20 \pm 0.05 \mathrm{kpc}$ at $1.5 \mathrm{GHz}$. However, the scale heights of the disk at $146 \mathrm{MHz}$ should be regarded with caution. The $146 \mathrm{MHz}$ emission in the inner disk is attenuated by free-free absorption which artificially increases the scale heights. Hence, we regard the systematic difference in the disk scale heights between $146 \mathrm{MHz}$ and $1.5 \mathrm{GHz}$ not to be significant.

The mean scale height of the halo emission is $2.30 \pm 0.70 \mathrm{kpc}$ at $146 \mathrm{MHz}$ compared to $1.36 \pm 0.19 \mathrm{kpc}$ at $1.5 \mathrm{GHz}$. The mean ratio $q_{\text {halo }}$ of scale heights of the halo emission between $146 \mathrm{MHz}$ and $1.5 \mathrm{GHz}$ (Table 6) is $1.7 \pm 0.3$. These results will be further discussed in Sect. 9.3.

The profiles of the nonthermal spectral index between $146 \mathrm{MHz}$ and $1.5 \mathrm{GHz}$ for different strips along the major axis (see Fig. 13) using the average intensities are shown in Fig. 15. The profiles show a consistently flat spectrum $(\approx-0.4)$ on the major axis of the galaxy and a roughly linear steepening with increasing distance from the major axis on both sides, reaching a spectral index of about -0.8 at $3 \mathrm{kpc}$ height.

The halo scale heights and spectral index profiles will be discussed in Sect. 9.3, using simplified models for the CRE propagation. A more thorough treatment, fitting the spectral index profiles with numerical models of CRE propagation such as of Heesen et al. (2016, 2018a) and Mulcahy et al. (2016) is deferred to future work.

\section{Discussion}

\subsection{Thermal absorption in the disk of NGC 891}

The total and nonthermal spectral index between $146 \mathrm{MHz}$ and $1.5 \mathrm{GHz}$ in the star-forming disk is observed to be significantly flatter than the injection spectrum $\left(\alpha_{\text {inj }} \simeq-0.6\right.$, see Sect. 6.4) and far flatter than what is observed at higher frequencies by Schmidt et al. (in prep.). Synchrotron self-absorption is unlikely to occur for such a diffuse source and is seen only for compact sources such as radio supernovae (Chevalier 1998). Bremsstrahlung losses of CREs dominate at low frequencies, but cannot flatten the radio spectrum (e.g. Basu et al. 2015). We propose that freefree absorption of nonthermal emission by ionized gas occurs in the disk.

Observations with the VLA at $330 \mathrm{MHz}$ and $74 \mathrm{MHz}$ suggested a flattening of the spectrum along the disk of the Milky Way (Kassim, private communication). Spectral turnovers due to free-free absorption have been observed in the integrated spectra of nearby starburst galaxies such M 82 at $\approx 500 \mathrm{MHz}$ (Adebahr et al. 2013), NGC 253 at $230 \mathrm{MHz}$ (Kapińska et al. 2017), and IC 10 at $322 \mathrm{MHz}$ (Basu et al. 2017). Spatially resolved free-free absorption has been observed in M 82 with Merlin at $408 \mathrm{MHz}$ (Wills et al. 1997) and with LOFAR at $154 \mathrm{MHz}$ (Varenius et al. 2015). Lacki \& Thompson (2010) showed that for M 82 free-free absorption from $\mathrm{H}_{\mathrm{II}}$ regions is the most important absorption process at frequencies down to $10 \mathrm{MHz}$. Low-frequency observations of several starburst galaxies with the Murchison Widefield Array (MWA) showed clear signs of free-free absorption in the spectrum of integrated flux densities (Galvin et al. 2018). Modelling of thermal absorption in nearby galaxies observed with the LOFAR Multifrequency Snapshot Sky Survey (MSSS) shows that absorption effects in non-starburst galaxies are visible in the integrated spectra only much below $100 \mathrm{MHz}$, but can be strong in the spectra of individual regions at higher frequencies, especially for edge-on galaxies (Chyży et al., in prep.).

While common in starburst galaxies, it is under debate if a flattening of the spectrum of integrated emission can be observed also in normal star-forming galaxies. From our NGC 891 data, we are able to study the spatially resolved distribution of free-free absorption in a highly inclined spiral galaxy for the first time. The distribution of nonthermal spectral index (Fig. 11) 
reveals regions in the disk with significant spectral flattening, while the spectrum of integrated emission (Fig. 9) shows a less obvious flattening towards lower frequencies, probably due to the contribution of steep-spectrum synchrotron emission from the galaxy's halo.

NGC 891 has a lower surface density of the star-formation rate compared with M 82 and the nuclear starburst of NGC 253, so that the space density of $\mathrm{H}_{\mathrm{II}}$ regions is lower in NGC 891; consequently, the emission measure (the square of the electron density integrated along the line of sight) and thus the spectral turnover frequency are lower as well. Furthermore, the emission measure and hence the turnover frequency vary for different lines of sight through the galaxy disk. As a result, any flattening of the integrated spectrum is smeared out over a large frequency range. This may explain why the integrated spectrum of NGC 891 around $146 \mathrm{MHz}$ hardly deviates from a power law.

To measure the possible flattening of the integrated nonthermal spectrum with higher accuracy, we first should ascertain the nature of the total emission spectrum of NGC 891. Since we are subtracting a simple optically thin free-free emission of NGC 891 throughout the radio frequency range studied here, any curvature in the total flux density spectrum itself will naturally give rise to a curvature in the nonthermal spectrum. A cursory look at the total flux densities in Fig. 9 (top panel) suggests that the measurements at the lowest frequencies, viz. 57.5 and $146 \mathrm{MHz}$, play an important role in determining the shape of the total intensity spectrum of NGC 891. New observations at similar frequencies, e.g. using the LOFAR Low Band Antenna (LBA) at around $55 \mathrm{MHz}$, could help us to ascertain whether the total intensity spectrum is indeed curved and thereby help to understand whether the curved nonthermal spectrum of NGC 891 is due to flattening at the lower frequencies or steepening at the higher frequencies. We performed fits to the total flux density spectrum by a power law, assuming varying values of the expected flux density at $55 \mathrm{MHz}$. If the LBA measures a flux density between 8 and $9 \mathrm{Jy}$ with less than about $10 \%$ uncertainty in flux density, one can confidently rule out a curvature in the total flux density spectrum of NGC 891.

While spatially resolved observations of free-free absorption in the disks of external galaxies are now feasible, the same effect hampers the estimate of the magnetic field strength. As mentioned in Sect. 7, the presence of free-free absorption leads to an underestimate of the nonthermal intensity at low frequencies and hence of the magnetic field strength. On the other hand, low-frequency observations still play an essential role in estimating the magnetic field strength in the halo because they are less affected by spectral ageing of the CREs.

High-resolution observations with the Giant Meterwave Radio Telescope (GMRT) and the LOFAR LBA are currently in progress and will help to constrain the optical depth of the ISM in the disk of galaxies. With NGC 891 being nearby and having an inclination angle of $\approx 90^{\circ}$ and thus a large pathlength, it is an ideal target for further studies of free-free absorption with high spatial resolution.

\subsection{Loss processes of CREs in the halo of NGC 891}

The maximum contribution to the synchrotron emission at the frequency $v$ comes from CREs at the energy:

$E_{v}=\sqrt{\left(\frac{v}{16 \mathrm{MHz}}\right)\left(\frac{B_{\perp}}{\mu \mathrm{G}}\right)^{-1}} \mathrm{GeV}$.
CREs emitting at $146 \mathrm{MHz}$ have an energy of $\lesssim 1.0 \mathrm{GeV}$ in the disk of NGC $891(B \gtrsim 10 \mu \mathrm{G}$, Sect. 7$)$ and $\simeq 1.1 \mathrm{GeV}$ in the halo $(B \simeq 7 \mu \mathrm{G})$.

The half-power lifetime of the observable synchrotronemitting CREs is:

$t_{\text {syn }}=8.35 \times 10^{9} \mathrm{yr}\left(\frac{E_{v}}{\mathrm{GeV}}\right)^{-1}\left(\frac{B_{\perp}}{\mu \mathrm{G}}\right)^{-2}$,

where $B_{\perp}$ is the magnetic field strength perpendicular to the line of sight (assuming an isotropic pitch angle distribution). For an isotropic turbulent field, $B_{\perp}=\sqrt{2 / 3} B$. In NGC 891, $t_{\text {syn }}$ varies between $\lesssim 1.2 \times 10^{8} \mathrm{yr}$ in the disk and $\simeq 1.9 \times 10^{8} \mathrm{yr}$ in the halo at $146 \mathrm{MHz}$.

CREs losing energy via inverse Compton (IC) radiation losses have a half-power lifetime of:

$t_{\mathrm{IC}}=3.55 \times 10^{8} \mathrm{yr}\left(\frac{E}{\mathrm{GeV}}\right)^{-1}\left(\frac{U_{\mathrm{ph}}}{10^{-12} \mathrm{erg} \mathrm{cm}^{-3}}\right)^{-1}$,

where $U_{\mathrm{ph}}$ is the total photon energy density. Heesen et al. (2014) found that the ratio between $t_{\mathrm{syn}}$ and $t_{\mathrm{IC}}$ (identical to the ratio between $U_{\mathrm{ph}}$ and the magnetic energy density) varies typically between $10 \%$ and $80 \%$ in normal star-forming galaxies, although it may be higher in galaxies undergoing a starburst. In NGC 891, this ratio is about $16 \%$, estimated from the total infrared luminosity and assuming energy equipartition (Heesen et al. 2018a), so that synchrotron losses dominate over IC losses.

The CRE half-power lifetime against bremsstrahlung losses is:

$t_{\text {brems }}=3.96 \times 10^{7} \mathrm{yr}\left(\frac{n}{\mathrm{~cm}^{-3}}\right)^{-1}$,

where $n$ is the density of the neutral gas. According to Basu et al. (2015), bremsstrahlung losses of CREs emitting in the range $100-300 \mathrm{MHz}$ dominate in regions with gas column densities beyond about $5 \mathrm{M}_{\odot} / \mathrm{pc}^{2}$, as is the case in the disk of NGC 891 .

The CRE half-power lifetime against ionization losses is (Longair 2011):

$t_{\text {ion }}=4.1 \times 10^{9} \mathrm{yr}\left(\frac{n}{\mathrm{~cm}^{-3}}\right)^{-1}\left(\frac{E}{\mathrm{GeV}}\right)\left[3 \ln \left(\frac{E}{\mathrm{GeV}}\right)+42.5\right]^{-1}$.

At frequencies below $300 \mathrm{MHz}$, ionization losses are slightly less important than bremsstrahlung losses (Basu et al. 2015), except perhaps in highly dense gas clouds.

\subsection{CRE propagation in NGC 891}

The scale heights of the nonthermal halo emission at $146 \mathrm{MHz}$ are consistently larger than those at $1.5 \mathrm{GHz}$ (Table 6). The probable reason is that CRE energy loss processes are weaker at lower frequencies, so that the CRE lifetime and hence the propagation height of CREs are larger.

We investigated two basic models of vertical CRE propagation, namely diffusion or advection (outflow) (Tabatabaei et al. 2013; Mulcahy et al. 2014, 2016; Krause et al. 2018). Mulcahy et al. (2016) showed via numerical modelling that diffusion is the dominant CRE propagation process in the disk of M 51. As the observed synchrotron spectral indices in the disk are too flat to be explained by dominating synchrotron losses, the CREs of M 51 must escape from the disk before losing their energy, e.g. by diffusion perpendicular to the disk. 

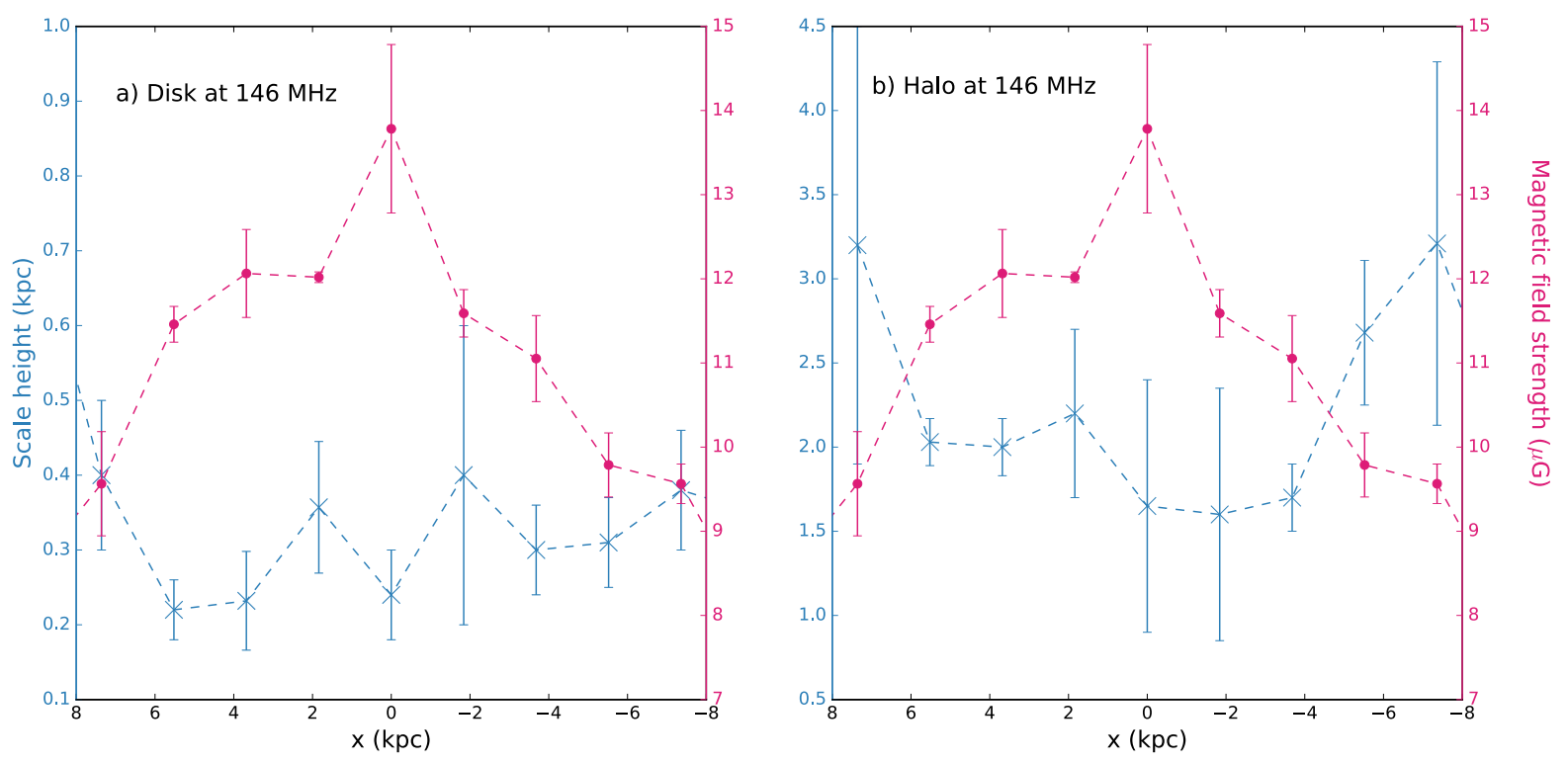

Fig. 16. Scale heights in kpc (dark blue) at $146 \mathrm{MHz}$ and magnetic field strengths in $\mu \mathrm{G}$ (red) at different distances $x$ from the galaxy's centre along the major axis (in kpc). $x>0$ corresponds to the north-eastern side of the galaxy and $x<0$ to the south-western side. The disk scale heights are shown in the left panel, the halo scale heights in the right panel. The magnetic field strengths are measured from the $1.5 \mathrm{GHz}$ data on the galaxy's major axis and are identical in all four panels.

We assume that the lifetime of CREs in the halo of NGC 891 is dominated by synchrotron loss (Eq. (3)), i.e. the halo is a calorimeter for CREs. In the case that radiation losses are important, a galaxy is referred to as an "electron calorimeter", where the CREs are losing their energy through radiation and ionization losses before they escape. Further assuming that the diffusion coefficient $D$ is constant (no dependencies on height $z$, nor on CRE energy $E$, nor on field strength $B$ ), the diffusive propagation height is:

$h_{\text {diff }}=\sqrt{D t_{\mathrm{CRE}}} \propto B^{-3 / 4} v^{-1 / 4}$

where $t_{\mathrm{CRE}}$ is the lifetime of the CREs (see Sect. 9.2) and $B$ is the field strength. With the frequency ratio between $146 \mathrm{MHz}$ and $1.5 \mathrm{GHz}$ of $1 / 10.3$, the expected ratio of propagation heights is 1.8. In case of dominating IC losses of the CREs, the frequency dependence of the diffusive propagation height $h_{\text {diff }}$ remains the same, while there is only a weak positive dependence on field strength $\left(h_{\text {diff }} \propto B^{1 / 4}\right)$.

For a constant advection speed $v$ and a lifetime of CRE dominated by synchrotron loss, the advective propagation height is:

$h_{\mathrm{adv}}=\mathrm{v} t_{\mathrm{CRE}} \propto B^{-3 / 2} v^{-1 / 2}$.

With the same frequency ratio as above, the expected ratio of propagation heights is 3.2. In the case of dominating IC losses of the CREs, the frequency dependence of the advective propagation height $h_{\mathrm{adv}}$ remains the same, while the dependence on field strength becomes positive $\left(h_{\mathrm{adv}} \propto B^{1 / 2}\right)$.

Several spiral galaxies in the CHANG-ES sample show indications for advective CRE propagation and CRE timescales dominated by escape from the halo (Krause et al. 2018). In this case, the propagation height does neither depend on frequency nor on magnetic field strength.

The mean observed ratio $q_{\text {halo }}$ of scale heights of the halo emission between $146 \mathrm{MHz}$ and $1.5 \mathrm{GHz}$ (Table 6) of $1.7 \pm$ 0.3 agrees well with the diffusion model and dominating synchrotron and/or IC losses. It seems that the CRE propagation from the disk to the halo of NGC 891 is slower than in other spiral edge-on galaxies in the CHANG-ES sample, so that the CRE synchrotron lifetime is smaller than the escape time.

The halo scale heights from Table 6 may suffer from various systematic bias effects. Firstly, the halo scale heights at $146 \mathrm{MHz}$ can be affected by free-free absorption in the disk. As a test, we artificially increased the nonthermal intensity in the inner disk by a factor of 3 . This leads to about $2 \times$ smaller scale heights of the inner disk, while the halo scale heights do not change within the limits of uncertainty.

NGC 891 was observed in L-band with the VLA D-array for $3 \times 10 \mathrm{~min}$. According to the VLA website, the maximum visible structure of a single snapshot observation at $1.5 \mathrm{GHz}$ is about $8^{\prime}$ which is similar to the angular extent of NGC 891. The maximum visible structure of a multiple snapshot observation is somewhat larger but hard to estimate. We cannot exclude that some diffuse halo emission is missing in the L-band image by Schmidt et al. (in prep.). The effect on the total flux density must be small because Fig. 9 does not indicate an obvious deficit, but the emission in the outer halo may still be underestimated, so that the measured halo scale heights could be too small. As a test, we added an artificial halo component which is constructed by smoothing the galaxy's image with a Gaussian function of $5^{\prime}$ full-width-at-half-maximum and decreasing the resulting intensities by a factor of 10 . Hence, this component contributes $10 \%$ to the total flux density which is still consistent with Fig. 9. It leads to an increase of the halo scale heights by only 5-10\% and to an insignificant decrease of the mean ratio of scale heights of the halo emission between $146 \mathrm{MHz}$ and $1.5 \mathrm{GHz}$ to 1.6 .

Both CRE propagation models discussed above are rather simplistic. A more detailed analysis needs a proper description of CRE sources, magnetic field distribution, CRE loss processes, and CRE propagation mechanisms. Such a study, based on the CHANG-ES data, is forthcoming (Schmidt et al. in prep.).

Nevertheless, we can explore the above models further, taking advantage of the fact that synchrotron emission is the only CRE loss process related to the magnetic field strength. Hence, the local scale height, as measured in the strips, should depend 
on the local magnetic field strength in the same area. We expect an increase of scale height with decreasing field strength which is different for diffusive and advective CRE propagation (Eqs. (7) and (8)).

The scale heights at $146 \mathrm{MHz}$ (Table 6) are plotted in Fig. 16. A variation of the disk scale height (left panel) with distance from the galaxy centre is not obvious, while the scale height of the halo (right panel) increases with increasing distance ("flaring"). This effect is similar to the one seen in the halo of the edge-on spiral galaxy NGC 253 (Heesen et al. 2009).

To investigate whether the halo flaring in NGC 891 is caused by a decrease of the synchrotron losses of CREs, we also show in Fig. 16 the magnetic field strengths on the major axis which are a measure of the level of synchrotron losses in the different strips (Eq. (3)). As we were unable to obtain reliable magnetic field strength estimates in the disk from our $146 \mathrm{MHz}$ data due to free-free absorption effects (see above), we instead use the magnetic field strength estimates from Schmidt et al. (in prep.) derived from $1.5 \mathrm{GHz}$ data. Indeed, the scale heights at both frequencies are smallest near to the centre where the field strengths are largest.

The relation between the scale heights at $146 \mathrm{MHz}$ and the magnetic field strength is shown in Fig. 17. The exponent of $-1.2 \pm 0.6$ is consistent with diffusive or advective propagation (Eqs. (7) and (8)) within the uncertainty. The negative sign of the exponents at both frequencies supports our above result that synchrotron radiation is the dominating loss process of CREs in the halo of NGC 891 and that IC losses are less important, which is generally the case for late-type spiral and dwarf irregular galaxies (Heesen et al. 2014).

The disk of NGC 891 seen with LOFAR is not uniform everywhere, but bulges out in the north where the halo scale height is large (Fig. 16, at $x=7.36 \mathrm{kpc}$ ), even larger than expected for the weak magnetic field in this area, as indicated by the points above the fit line in the top left corners of Fig. 17. Possible reasons could be a larger diffusion coefficient in the northern star-forming region or an increase of scale height by an enhanced, faster outflow.

According to Strong (1978), the vertical profile of the nonthermal spectral index is similar for the two types of CRE propagation, diffusion and advection. On the other hand, Heesen et al. (2016, 2018a) showed that the vertical profile of the radio spectral index can help to distinguish between the two basic mechanisms of CRE transport. Advection of CREs with a constant outflow velocity should lead to linear profiles of the nonthermal spectral index, where the spectral index steepens gradually with increasing distance from the disk plane. Conversely, diffusion should lead to parabolic-shaped spectral index profiles where the spectral index remains about constant until a few kpc height, from where on the synchrotron losses become strong and the nonthermal spectral index steepens rapidly. In NGC 891, the vertical profiles of the radio spectral index between $146 \mathrm{MHz}$ and $1.5 \mathrm{GHz}$, as presented in Fig. 15, are falling into the first category, indicating that advection in an outflow may be the dominating CRE transport process. In non-calorimetric halos where CRE escape is important, the nonthermal scale height and CRE lifetime are not related, and Eqs. (7) and (8) do not apply.

In order to perform a more quantitative investigation, we need an estimate of the escape timescale for the cosmic rays in the halo of NGC 891 and hence a model of the outflow velocity as a function of distance from the disk. Based on previous radio data, Heesen et al. (2018a) could not find a wellconstrained advection speed for a possible outflow in NGC 891.

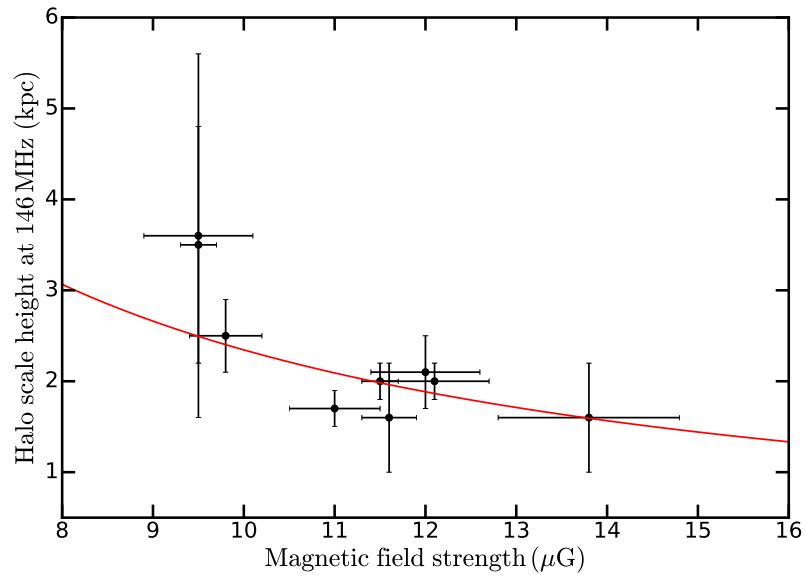

Fig. 17. Halo scale heights at $146 \mathrm{MHz}$ in the 9 strips along the major axis of NGC 891 as a function of magnetic field strengths on the major axis. The red line shows the best-fit exponential function.

We plan to combine our new LOFAR $146 \mathrm{MHz}$ data of NGC 891 and the CHANG-ES data at 1.5 and $6 \mathrm{GHz}$ in a forthcoming work. An analysis of the profiles of synchrotron emission and spectral index will be performed, where the 1D diffusionloss equation will be solved, such as has been done for the radial diffusion within the disk of the almost face-on galaxy M51 (Mulcahy et al. 2016) and for the vertical transport by advection and diffusion in the halos of many edge-on galaxies (Heesen et al. 2016, 2018a). This will allow us to measure diffusion coefficients or advection speeds of CREs in the halo of NGC 891.

\section{Conclusions}

In this work, we performed radio continuum observations of the edge-on spiral galaxy NGC 891 with the LOFAR High Band Antenna (HBA) Array with a central frequency of $146 \mathrm{MHz}$ and with the Arcminute Microkelvin Imager (AMI) at $15.5 \mathrm{GHz}$. Using the facet calibration scheme detailed in van Weeren et al. (2016), we achieved LOFAR images with a high quality near to thermal noise which provide a sensitive view of the extended halo of NGC 891 and its physical origin. This is the first time that low-frequency, high-resolution observations are presented of this well-studied edge-on galaxy.

We used the AMI Small and Large Array to observe NGC 891 at a central frequency of $15.5 \mathrm{GHz}$. For the first time, a nearby galaxy has been studied using the upgraded correlator of AMI. Nearby galaxies have rarely been observed at radio frequencies larger than $10 \mathrm{GHz}$. This work paves the way for an AMI nearby galaxy survey at $15.5 \mathrm{GHz}$.

The main findings of this work are:

- With our new measurements and assuming realistic uncertainties of previous measurements, we derived the spectrum of the integrated nonthermal flux density. The spectrum is probably not a simple power law. It can be fitted by a power law with a steepening in spectral index by -0.5 towards higher frequencies, or by a curved polynomial, indicating a flattening towards lower frequencies by free-free absorption and a steepening towards higher frequencies. Which physical mechanism produces the curvature in the spectrum cannot be constrained with the current measurements. A clear detection of a spectral curvature in the integrated spectrum needs observations with LOFAR LBA at around $55 \mathrm{MHz}$ with $<10 \%$ uncertainty. 


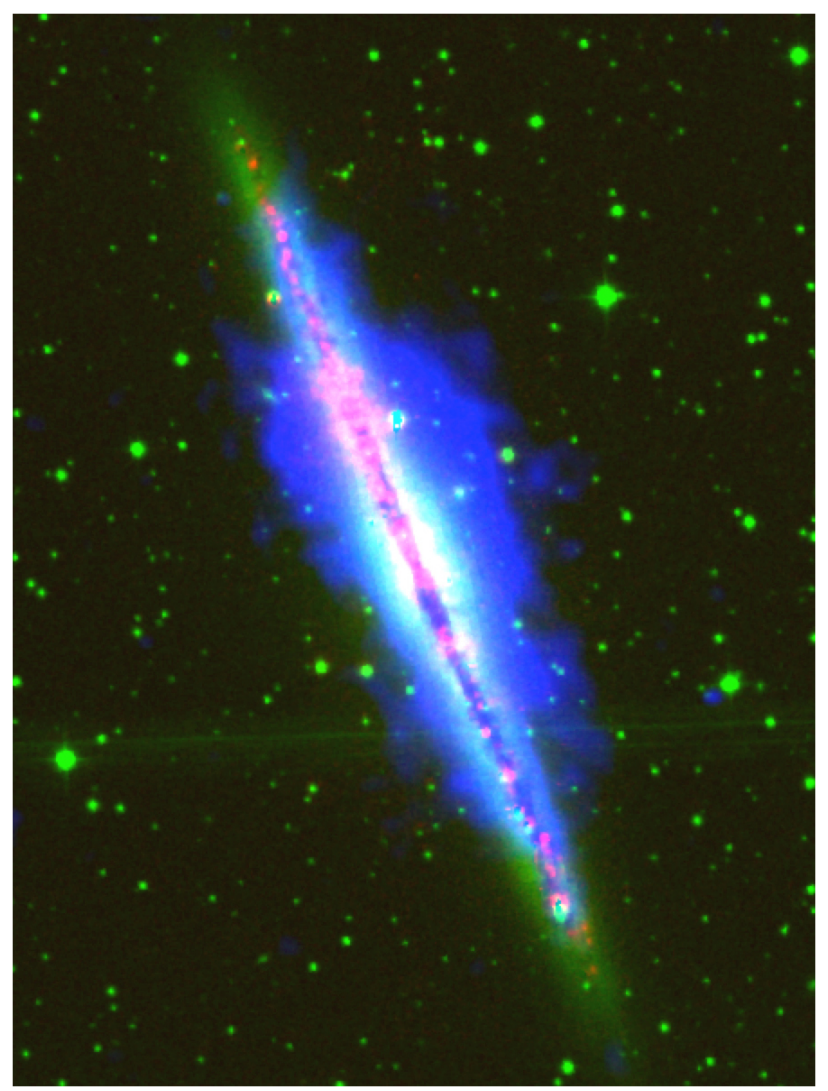

Fig. 18. Composite of NGC 891: LOFAR $146 \mathrm{MHz}$ at $12^{\prime \prime} \times 12^{\prime \prime}$ resolution (blue), optical from DSS2 (green), and $\mathrm{H} \alpha$ from Rand et al. $\left(1990 ;\right.$ red). The size of the image is $9^{\prime} \times 12^{\prime}$.

- We find no significant flattening of the spectrum by thermal emission towards higher frequencies of up to $15.5 \mathrm{GHz}$, suggesting that the synchrotron component is still dominant in this frequency range. Further observations at higher frequencies are necessary to confirm this result.

- In the star-forming disk, our map of nonthermal spectral index between $146 \mathrm{MHz}$ and the CHANG-ES data at $1.5 \mathrm{GHz}$ reveals areas with significant spectral flattening towards lower frequencies, with values significantly flatter than -0.5 . This is likely caused by free-free absorption in the ionized gas.

- In the halo, we observe nonthermal spectral indices between $146 \mathrm{MHz}$ and $1.5 \mathrm{GHz}$ within a range of -0.6 to -0.8 , significantly flatter than the spectral indices at higher frequencies. This supports the expectation that cosmic-ray electrons (CREs) emitting at low frequencies suffer less from energy losses. Consequently, low-frequency observations are better suited to estimate the magnetic field in the halo if one assumes energy equipartition between cosmic rays and magnetic fields.

- The mean magnetic field strength in the halo is $7 \pm 2 \mu \mathrm{G}$. Due to the significant free-free absorption, we cannot confidently estimate magnetic field strengths in the disk of NGC 891. High-frequency observations are more suited for this task, while low-frequency observations are more suitable to estimate magnetic field strengths in the halo.

- Radio emission from the halo at $146 \mathrm{MHz}$ is detected out to a maximum of $7.3 \mathrm{kpc}$ distance from the major axis. The similar extension compared to the new CHANG-ES image at $1.5 \mathrm{GHz}$ is most likely due to the lower sensitivity at $146 \mathrm{MHz}$.
- The scale heights of the nonthermal halo emission at $146 \mathrm{MHz}$ are consistently larger at all radii than those at $1.5 \mathrm{GHz}$, with a mean ratio of $1.7 \pm 0.3$, as predicted by diffusive CRE propagation. This ratio also suggests that spectral ageing of the CREs, caused by radiation losses, is important and hence that the halo is at least a partial calorimeter for CREs, i.e. they are losing a significant fraction of their energy before they can escape.

- The scale height of the nonthermal halo emission at $146 \mathrm{MHz}$ correlates with the magnetic field strength with an exponent of $-1.2 \pm 0.6$, consistent with diffusive or advective CRE propagation. The negative exponent is a signature of dominating synchrotron losses in the halo. IC losses appear to be less important.

- The low-frequency radio halo in NGC891 seems to be different from the ones observed in the CHANG-ES sample at $\mathrm{GHz}$ frequencies that are escape-dominated (i.e. noncalorimetric). More low-frequency observations of edge-on spiral galaxies are needed in order to decide whether this is a specific property of the low-frequency halo in NGC 891 or holds true for spiral galaxies in general.

- The linearly steepening spectral index profiles of the nonthermal emission in the halo seem to favour advection of CREs in a galactic wind (Heesen et al. 2016). Further studies are forthcoming, using refined modelling of CRE propagation and exploiting the combined LOFAR and CHANG-ES data at 1.5 and $6 \mathrm{GHz}$.

- To measure energy losses and propagation of CREs and magnetic field strengths in the disk and halo with higher accuracy, further observations of NGC 891 and other edgeon spiral galaxies over a wide frequency coverage and with high spatial resolution are needed.

Acknowledgements. This research was performed in the framework of the DFG Research Unit 1254 "Magnetisation of Interstellar and Intergalactic Media: The Prospects of Low-Frequency Radio Observations". LOFAR, designed and constructed by ASTRON, has facilities in several countries that are owned by various parties (each with their own funding sources) and that are collectively operated by the International LOFAR Telescope (ILT) foundation under a joint scientific policy. We acknowledge support by the FZ Jülich under Jureca computing grant HTB00. We thank the staff of the Mullard Radio Astronomy Observatory for their invaluable assistance in the commissioning and operation of AMI which is supported by Cambridge and Oxford Universities. We acknowledge support from the European Research Council under grant ERC-2012-StG-307215 LODESTONE. This research made use of the NASA/IPAC Extragalactic Database (NED) which is operated by the Jet Propulsion Laboratory, California Institute of Technology, under contract with the National Aeronautics and Space Administration. A.B. acknowledges financial support by the German Federal Ministry of Education and Research (BMBF) under grant 05A17PB1 (Verbundprojekt DMeerKAT). F.S.T. acknowledges financial support from the Spanish Ministry of Economy and Competitiveness (MINECO) under grant AYA2016-76219-P. We thank Frank Israel (Sterrewacht Leiden) and Olaf Wucknitz (MPIfR Bonn) for carefully reading of the manuscript and our anonymous referee for many useful comments.

\section{References}

Adebahr, B., Krause, M., Klein, U., et al. 2013, A\&A, 555, A23 Allen, R. J., Sancisi, R., \& Baldwin, J. E. 1978, A\&A, 62, 397 Alton, P. B., Bianchi, S., Rand, R. J., et al. 1998, ApJ, 507, L125 Arshakian, T. G., Stepanov, R., Beck, R., Krause, M., \& Sokoloff, D. 2011, Astron. Nachr., 332, 524

Baars, J. W. M., Genzel, R., Pauliny-Toth, I. I. K., \& Witzel, A. 1977, A\&A, 61, 99

Baldwin, J. E., \& Pooley, G. G. 1973, MNRAS, 161, 127

Basu, A., Beck, R., Schmidt, P., \& Roy, S. 2015, MNRAS, 449, 3879

Basu, A., Roychowdhury, S., Heesen, V., et al. 2017, MNRAS, 471, 337

Beck, R. 2015, A\&ARv, 24, 4

Beck, R., Biermann, P., Emerson, D. T., \& Wielebinski, R. 1979, A\&A, 77, 25 
Beck, R., Brandenburg, A., Moss, D., Shukurov, A., \& Sokoloff, D. 1996, ARA\&A, 34, 155

Beck, R., \& Krause, M. 2005, Astron. Nachr., 326, 414

Bell, A. R. 1978, MNRAS, 182, 443

Bietenholz, M. F., \& Bartel, N. 2017, ApJ, 851, 7

Bietenholz, M. F., Bartel, N., \& Rupen, M. P. 2002, ApJ, 581, 1132

Bietenholz, M. F., Bartel, N., \& Rupen, M. P. 2010, ApJ, 712, 1057

Bregman, J. N., \& Houck, J. C. 1997, ApJ, 485, 159

Bregman, J. N., \& Pildis, R. A. 1994, ApJ, 420, 570

Buffie, K., Heesen, V., \& Shalchi, A. 2013, ApJ, 764, 37

Caprioli, D. 2011, J. Cosmol. Astropart. Phys., 5, 026

Chevalier, R. A. 1998, ApJ, 499, 810

Condon, J. J. 1987, ApJS, 65, 485

Cornwell, T. J. 2008, IEEE J. Select. Top. Signal Process., 2, 793

Dahlem, M., Dettmar, R.-J., \& Hummel, E. 1994, A\&A, 290, 384

de Jong M. L. 1967, ApJ, 150, 1

de Vaucouleurs, G., de Vaucouleurs, A., Corwin Jr., H. G., et al. 1991, Third Reference Catalogue of Bright Galaxies (New York: Springer)

Dettmar, R.-J. 1990, A\&A, 232, L15

Dumke, M., Krause, M., Wielebinski, R., \& Klein, U. 1995, A\&A, 302, 691

Ekers, R. D., \& Sancisi, R. 1977, A\&A, 54, 973

Elstner, D., Golla, G., Rüdiger, G., \& Wielebinski, R. 1995, A\&A, 297, 77

Fraternali, F. 2017, in Astrophysics and Space Science Library, Gas Accretion onto Galaxies, eds. A. Fox, \& R. Davé, 430, 323

Galvin, T. J., Seymour, N., Marvil, J., et al. 2018, MNRAS, 474, 779

Garcia-Burillo, S., Guelin, M., Cernicharo, J., \& Dahlem, M. 1992, A\&A, 266, 21

Gioia, I. M., \& Fabbiano, G. 1987, ApJS, 63, 771

Gioia, I. M., \& Gregorini, L. 1980, A\&AS, 41, 329

Gioia, I. M., Gregorini, L., \& Klein, U. 1982, A\&A, 116, 164

Green, D. A. 2014, Bull. Astronom. Soc. India, 42, 47

Gregory, P. C., \& Condon, J. J. 1991, ApJS, 75, 1011

Heeschen, D. S., \& Wade, C. M. 1964, AJ, 69, 277

Heesen, V., Beck, R., Krause, M., \& Dettmar, R.-J. 2009, A\&A, 494, 563

Heesen, V., Brinks, E., Leroy, A. K., et al. 2014, AJ, 147, 103

Heesen, V., Dettmar, R.-J., Krause, M., Beck, R., \& Stein, Y. 2016, MNRAS, 458,332

Heesen, V., Krause, M., Beck, R., et al. 2018a, MNRAS, 476, 158

Heesen, V., Rafferty, D. A., Horneffer, A., et al. 2018b, MNRAS, 476, 1756

Henriksen, R. N., \& Irwin, J. A. 2016, MNRAS, 458, 4210

Hickish, J., Razavi-Ghods, N., Perrott, Y. C., et al. 2018, MNRAS, 475, 5677

Hodges-Kluck, E. J., \& Bregman, J. N. 2013, ApJ, 762, 12

Högbom, J. A. 1974, A\&AS, 15, 417

Howk, J. C., \& Savage, B. D. 1997, AJ, 114, 2463

Hummel, E. 1991, A\&A, 251, 442

Hummel, E., Dahlem, M., van der Hulst, J. M., \& Sukumar, S. 1991, A\&A, 246, 10

Irwin, J., Beck, R., Benjamin, R. A., et al. 2012, AJ, 144, 43

Israel, F. P., \& van der Hulst, J. M. 1983, AJ, 88, 1736

Israel, F. P., \& Mahoney, M. J. 1990, ApJ, 352, 30

Israel, F. P., van der Werf, P. P., \& Tilanus, R. P. J. 1999, A\&A, 344, L83

Kapińska, A. D., Staveley-Smith, L., Crocker, R., et al. 2017, ApJ, 838, 68

Kazès, I., Le Squeren, A. M., \& Nguyen-Quang-Rieu. 1970, Astrophys. Lett., 6, 193

Kellermann, K. I., Pauliny-Toth, I. I. K., \& Williams, P. J. S. 1969, ApJ, 157, 1

Klein, U., Wielebinski, R., \& Beck, R. 1984, A\&A, 133, 19

Klein, U., Lisenfeld, U., \& Verley, S. 2018, A\&A, 611, A55

Krause, M. 2009, Rev. Mex. Astron. Astrofis., 36, 25

Krause, M. 2012, in Magnetic Fields in the Universe III - From Laboratory and Stars to Primordial Structures, eds. M. Soida, K. Otmianowska-Mazur, E. M. de Gouveia Dal Pino, \& A. Lazarian, (Krakow: Jagiellonian University) 155

Krause, M., Irwin, J., Wiegert, T., et al. 2018, A\&A, 611, A72

Kulsrud, R., \& Pearce, W. P. 1969, ApJ, 156, 445

Lacki, B. C., \& Thompson, T. A. 2010, ApJ, 717, 196

Lepage, R., \& Billard, L. 1992, Exploring the Limits of Bootstrap, Wiley Series in Probability and Mathematical Statistics (New York: Wiley)

Lerche, I., \& Schlickeiser, R. 1981, ApJS, 47, 33

Lerche, I., \& Schlickeiser, R. 1982, A\&A, 107, 148
Licquia, T., \& Newman, J. 2014, in Amer. Astron. Soc. Meet. Abstr., 223, 336.04 Longair, M. 2011, High Energy Astrophysics, 3rd ed. (Cambridge, UK: Cambridge University Press)

Marvil, J., Owen, F., \& Eilek, J. 2015, AJ, 149, 32

McMullin, J. P., Waters, B., Schiebel, D., Young, W., \& Golap, K. 2007, in Astronomical Data Analysis Software and Systems XVI, eds. R. A. Shaw, F. Hill, \& D. J. Bell, ASP Conf. Ser., 376, 127

Moss, D., \& Sokoloff, D. 2017, A\&A, 598, A72

Moss, D., Sokoloff, D., Beck, R., \& Krause, M. 2010, A\&A, 512, A61

Mulcahy, D. D., Fletcher, A., Beck, R., Mitra, D., \& Scaife, A. M. M. 2016, A\&A, 592, A123

Mulcahy, D. D., Horneffer, A., Beck, R., et al. 2014, A\&A, 568, A74

Müller, P., Krause, M., Beck, R., \& Schmidt, P. 2017, A\&A, 606, A41

Niklas, S., Klein, U., \& Wielebinski, R. 1997, A\&A, 322, 19

Nixon, C. J., Hands, T. O., King, A. R., \& Pringle, J. E. 2018, MNRAS, 477, 3539

Norman, C. A., \& Ikeuchi, S. 1989, ApJ, 345, 372

Offringa, A. R., de Bruyn, A. G., Biehl, M., et al. 2010, MNRAS, 405, 155

Offringa, A. R., van de Gronde, J. J., \& Roerdink, J. B. T. M. 2012, A\&A, 539, A95

Okabe, A., Boots, B., Sugihara, K., \& Chiu, S. 2000, Spatial tessellations. Concepts and Applications of Voronoi Diagrams: Wiley Series in Probability and Mathematical Statistics, 2nd ed.

Oosterloo, T., Fraternali, F., \& Sancisi, R. 2007, AJ, 134, 1019

Pakmor, R., Marinacci, F., \& Springel, V. 2014, ApJ, 783, L20

Perley, R. A., \& Butler, B. J. 2013, ApJS, 204, 19

Pohl, M., \& Schlickeiser, R. 1990, A\&A, 234, 147

Rand, R. J., Kulkarni, S. R., \& Hester, J. J. 1990, ApJ, 352, L1

Rau, U., \& Cornwell, T. J. 2011, A\&A, 532, A71

Rengelink, R. B., Tang, Y., de Bruyn, A. G., et al. 1997, A\&AS, 124, 259

Rossa, J., Dettmar, R.-J., Walterbos, R. A. M., \& Norman, C. A. 2004 AJ, 128,674

Rupen, M. P. 1991, AJ, 102, 48

Scaife, A. M. M., \& Heald, G. H. 2012, MNRAS, 423, L30

Schmidt, P. 2016, PhD Thesis, University of Bonn, Germany

Scoville, N. Z., Thakkar, D., Carlstrom, J. E., \& Sargent, A. I. 1993, ApJ, 404, L59

Shapiro, P. R., \& Field, G. B. 1976, ApJ, 205, 762

Sofue, Y. 1987, PASJ, 39, 547

Soida, M., Krause, M., Dettmar, R.-J., \& Urbanik, M. 2011, A\&A, 531, A127

Stil, J. M., Krause, M., Beck, R., \& Taylor, A. R. 2009, ApJ, 693, 1392

Strickland, D. K., Heckman, T. M., Colbert, E. J. M., Hoopes, C. G., \& Weaver, K. A. 2004, ApJS, 151, 193

Strong, A. W. 1978, A\&A, 66, 205

Tabatabaei, F. S., Beck, R., Krügel, E., et al. 2007, A\&A, 475, 133

Tabatabaei, F. S., Berkhuijsen, E. M., Frick, P., Beck, R., \& Schinnerer, E. 2013, A\&A, 557, A129

Tabatabaei, F. S., Schinnerer, E., Krause, M., et al. 2017, ApJ, 836, 185

Temple, R. F., Raychaudhury, S., \& Stevens, I. R. 2005, MNRAS, 362, 581

Uhlig, M., Pfrommer, C., Sharma, M., et al. 2012, MNRAS, 423, 2374

van der Kruit, P. C., \& Searle, L. 1981, A\&A, 95, 116

van Gorkom, J., Rupen, M., Knapp, G., et al. 1986, IAU Circ., 4248, 1

van Haarlem, M. P., Wise, M. W., Gunst, A. W., et al. 2013, A\&A, 556, A2

van Weeren, R. J., Williams, W. L., Hardcastle, M. J., et al. 2016, ApJS, 223, 2

Varenius, E., Conway, J. E., Martí-Vidal, I., et al. 2015, A\&A, 574, A114

Vargas, C. J., Mora-Partiarroyo, S. C., Schmidt, P., et al. 2018, ApJ, 853, 128

Vigotti, M., Grueff, G., Perley, R., Clark, B. G., \& Bridle, A. H. 1989, AJ, 98, 419

Whaley, C. H., Irwin, J. A., Madden, S. C., Galliano, F., \& Bendo, G. J. 2009, MNRAS, 395, 97

Wiegert, T., Irwin, J., Miskolczi, A., et al. 2015, AJ, 150, 81

Williams, W. L., van Weeren, R. J., Röttgering, H. J. A., et al. 2016, MNRAS, 460, 2385

Wills, K. A., Pedlar, A., Muxlow, T. W. B., \& Wilkinson, P. N. 1997, MNRAS, 291, 517

Xilouris, E. M., Byun, Y. I., Kylafis, N. D., Paleologou, E. V., \& Papamastorakis, J. 1999, A\&A, 344, 868

Zwart, J. T. L., Barker, R. W., Biddulph, P., et al. 2008, MNRAS, 391, 1545 